ARTICLE

\title{
Systemic muscle wasting and coordinated tumour response drive tumourigenesis
}

Holly Newton (1) 1,2, Yi-Fang Wang 1,2, Laura Camplese ${ }^{1,2}$, Joao B. Mokochinski (1) 1,2, Holger B. Kramer (1D 1,2,

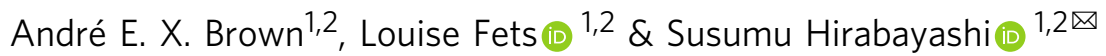

Cancer cells demand excess nutrients to support their proliferation, but how tumours exploit extracellular amino acids during systemic metabolic perturbations remain incompletely understood. Here, we use a Drosophila model of high-sugar diet (HSD)-enhanced tumourigenesis to uncover a systemic host-tumour metabolic circuit that supports tumour growth. We demonstrate coordinate induction of systemic muscle wasting with tumour-autonomous Yorkie-mediated SLC36-family amino acid transporter expression as a proline-scavenging programme to drive tumourigenesis. We identify Indole-3-propionic acid as an optimal amino acid derivative to rationally target the proline-dependency of tumour growth. Insights from this whole-animal Drosophila model provide a powerful approach towards the identification and therapeutic exploitation of the amino acid vulnerabilities of tumourigenesis in the context of a perturbed systemic metabolic network.

\footnotetext{
${ }^{1}$ Medical Research Council London Institute of Medical Sciences, Du Cane Road, London W12 0NN, UK. ${ }^{2}$ Institute of Clinical Sciences, Faculty of Medicine,

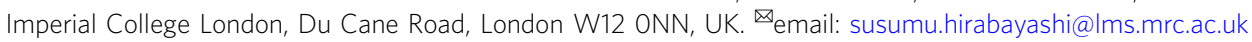


C ancer cells require a constant supply of metabolic intermediates to support their proliferation. To meet the biosynthetic demands associated with tumourigenesis, cancer cells actively acquire nutrients from the extracellular space ${ }^{1-5}$. Cancer is a systemic disease that associates with a range of host metabolic abnormalities such as obesity, insulin resistance and cancer-associated cachexia; each of which alters the host systemic nutritional environment. These changes in both nutrient composition and availability may have profound effects on cancer development and progression. However, how cancer cells sense and respond to nutritional changes in the context of organismal metabolic alterations remains an underexplored area in cancer biology.

Cancer-associated cachexia is a systemic metabolic syndrome of weight loss associated with progressive skeletal muscle wasting 6,7 . The multifactorial and heterogeneous condition of cachexia involves a complex multi-organ interplay, which has impeded its comprehensive understanding at the molecular level ${ }^{8}$. A series of recent studies using Drosophila melanogaster have shown that tumour-derived factors modulate host metabo$\operatorname{lism}^{9-11}$. In addition, tumour-derived factors promote the release of nutrients from the tumour microenvironment to promote tumour growth ${ }^{12}$. Here we leverage a Drosophila model of highsugar diet (HSD)-enhanced tumourigenesis and demonstrate that HSD-enhanced tumours induce SLC36-family transporter expression as a coordinated mechanism to exploit exogenous proline for tumourigenesis during systemic muscle wasting. Furthermore, we use these mechanistic insights to rationally target the proline dependency of tumours as an approach to inhibit tumour growth.

\section{Results}

Ras/Src-tumours promote muscle wasting in HSD. We previously reported a Drosophila larval model to study the systemic effects of HSD-induced obesity and insulin resistance on tumour progression ${ }^{13,14}$. Feeding Drosophila larvae an HSD led to sugardependent metabolic defects including accumulation of fat, systemic insulin resistance and hyperglycaemia ${ }^{15}$. Targeted coactivation of Ras- and Src-pathways in the Drosophila eye epithelia (Ras/Src-activated tumours)-by expression of an oncogenic isoform of $d R a s 1, \quad r a s 1^{G 12 V}$ and knockout of the negative regulator of Src, C-terminal src kinase $\left(c s k^{-/}\right)$-led to the development of benign tumours in animals raised on a control diet (CD) (Fig. 1a). Comparatively, feeding ras1 ${ }^{G 12 V} ; c s k^{-/-}$ animals an HSD promoted aggressive tumour growth in the eye epithelia (Fig. 1b and Supplementary Fig. 1a), associated with secondary tumour formation (Fig. 1b, arrowheads), and led to larval lethality (Supplementary Fig. 1b).

To explore the interplay between HSD-induced obesity, tumour progression and systemic organ wasting, we assessed the peripheral tissues of $\mathrm{rasl}^{\mathrm{G12V}} ; \mathrm{csk} \mathrm{K}^{-1-}$ animals-including the larval fat body and body-wall skeletal muscle. Fat body lipid accumulation was elevated under HSD feeding, as assessed by the neutral lipid stain Nile Red (Fig. 1c, d). Strikingly, despite possessing an intact fat body, $\operatorname{ras}^{\mathrm{G} 12 \mathrm{~V}} ; \mathrm{cs} \mathrm{k}^{-/-}$animals raised on HSD exhibited significant skeletal muscle wasting as visualised by F-actin and myosin staining (Fig. 1e, f and Supplementary Fig. 1c, d). To quantify the severity of muscle wasting-as assessed by imaging the fourth abdominal segment of the larval body-wall muscle (Supplementary Fig. 1e)-we scored animals into one of four categories: none, minor, moderate or strong wasting (Supplementary Fig. 1f-i). While $83.3 \%$ of the $\operatorname{ras}^{G 12 V} ;$ csk $^{-/-}$ animals raised on a CD displayed intact skeletal muscle, most of the animals raised on an HSD exhibited muscle wasting of some degree (Fig. 1m). Muscle wasting was progressive; as HSD- enhanced tumourigenesis proceeded, skeletal muscle wasting increased (Supplementary Fig. 1j-n). Functional locomotion defects-as measured by video tracking analysis ${ }^{16,17}$-were observed in ras1 $1^{G 12 V} ; c s k^{-/-}$animals raised on an HSD (Supplementary Fig. 1o, p).

Obesity and insulin resistance is an independent factor that can promote muscle wasting ${ }^{18}$. HSD feeding promoted lipid accumulation in tumour-free LacZ-animals, and also led to muscle wasting, albeit far weaker than $\operatorname{rasl}^{G 12 V}, c s k^{-/-}$animals in HSD (Fig. 1g-m). These results indicate that HSD-induced obesity induces systemic muscle wasting, and the presence of tumours further promotes muscle wasting.

To examine the possibility that observed muscle wasting was due to the ecdysone-mediated metamorphic process, we used the temperature-sensitive ecdysoneless mutant allele $\left(e c d^{1}\right)^{19}$. Tumour-free and $\operatorname{ras}^{G 12 V_{;}}$csk ${ }^{-1-}$ animals raised on HSD, exhibited muscle wasting of similar strength, regardless of whether they lacked ecdysoneless $\left(\mathrm{ecd}^{-/-}\right)$, indicating that muscle wasting occurred independently of ecdysone-mediated histolysis (Supplementary Fig. 2a-e). A blue dye feeding assay indicated that $\mathrm{ras} 1^{\mathrm{G12V}} ; \mathrm{csk} \mathrm{K}^{-1-}$ animals continue to feed in HSD (Supplementary Fig. $2 \mathrm{f}-\mathrm{k}$ ) and failure to detect leakage of blue dye from the gut lumen suggested intact gut integrity (Supplementary Fig. 2l-n). The morphological changes in salivary gland and midgut that occur during metamorphosis ${ }^{20}$ were not observed in HSD-fed ras $1^{G 12 V}$; $c s k^{-/}$animals (Supplementary Fig. 2o-t). Collectively, these results indicate that the metamorphic process does not account for the muscle wasting observed in ras $1^{G 12 V} ; c s k^{-/-}$animals in HSD, and instead support a specific role for HSD feeding and tumourigenesis in the promotion of muscle wasting.

Tumour-derived branchless mediates muscle wasting. We next set out to identify tumour-derived factors that promote muscle wasting in HSD. Through genome-wide transcriptional profiling analysis of dissected tumour tissue from $\mathrm{ras}^{\mathrm{G} 12 \mathrm{~V}} ; \mathrm{csk} \mathrm{k}^{-/-}$animals raised on CD and HSD (Supplementary Fig. 3a), we identified branchless (bnl), a Drosophila fibroblast growth factor (FGF) ligand as one of the most highly upregulated secreted factors in ras $1^{G 12 V}$;sk ${ }^{-/-}$tumours in animals raised on an HSD (upregulated 5.0- $\log 2$ fold) (Fig. 2a and Supplementary Fig. 3b). Among the three known Drosophila FGF ligands (Bnl, Pyramus and Thisbe), bnl was the most highly elevated FGF ligand in $\operatorname{rasl}^{G 12 V}$; $c s k^{-1-}$ tumours in HSD (Fig. 2a). Our previous study demonstrated that Bnl protein expression was elevated in the secondary tumours associated with tracheal branches ${ }^{13}$; we now demonstrate that Bnl protein expression is strongly upregulated in ras $1^{G 12 V_{;}} ; s k^{-/-}$primary tumours, but not in the wild-type (LacZ) eye discs of animals fed an HSD (Fig. 2b, c and Supplementary Fig. $3 c-e)$.

Reducing $b n l$ expression in Ras/Src-activated tumours $\left(\operatorname{ras} 1^{G 12 V} ; c s k^{-/-}, b n l^{R N A i}\right)$ attenuated muscle wasting in animals raised on an HSD (Fig. 2g, h, k and Supplementary Fig. 3f, g, q), indicating that tumour-derived $\mathrm{Bnl}$ is required for muscle wasting. Tumour-derived factors recently implicated in cachexia-like organ wasting include ImpL2 and $\mathrm{Pvfl}^{9-11}$. However, reducing the levels of either ImpL2 $\left(\mathrm{rasl}^{\mathrm{G} 12 \mathrm{~V}} ; \mathrm{csk} \mathrm{K}^{-/-}\right.$, ImpL2 $2^{R N A i}$ ) or Pvf1 (ras1 ${ }^{G 12 V}, p v f 1^{R N A i} ; c s k^{-/-}$) failed to rescue muscle wasting as efficiently as $b n l$ in our HSD-enhanced Ras/ Src-tumour model (Supplementary Fig. 3h-k, q). Similarly, reducing the expression of Pvf2 (ras1 $\left.{ }^{G 12 V} ; c s k^{-/}, p v f 2^{R N A i}\right)$ did not rescue muscle wasting (Supplementary Fig. 3l, m, q). Ectopic expression of $b n l$ from the fat body promoted muscle wasting in HSD, indicating that systemic Bnl promotes muscle wasting (Fig. 2i-k). Altogether, these results demonstrate $\mathrm{Bnl}$ as a 

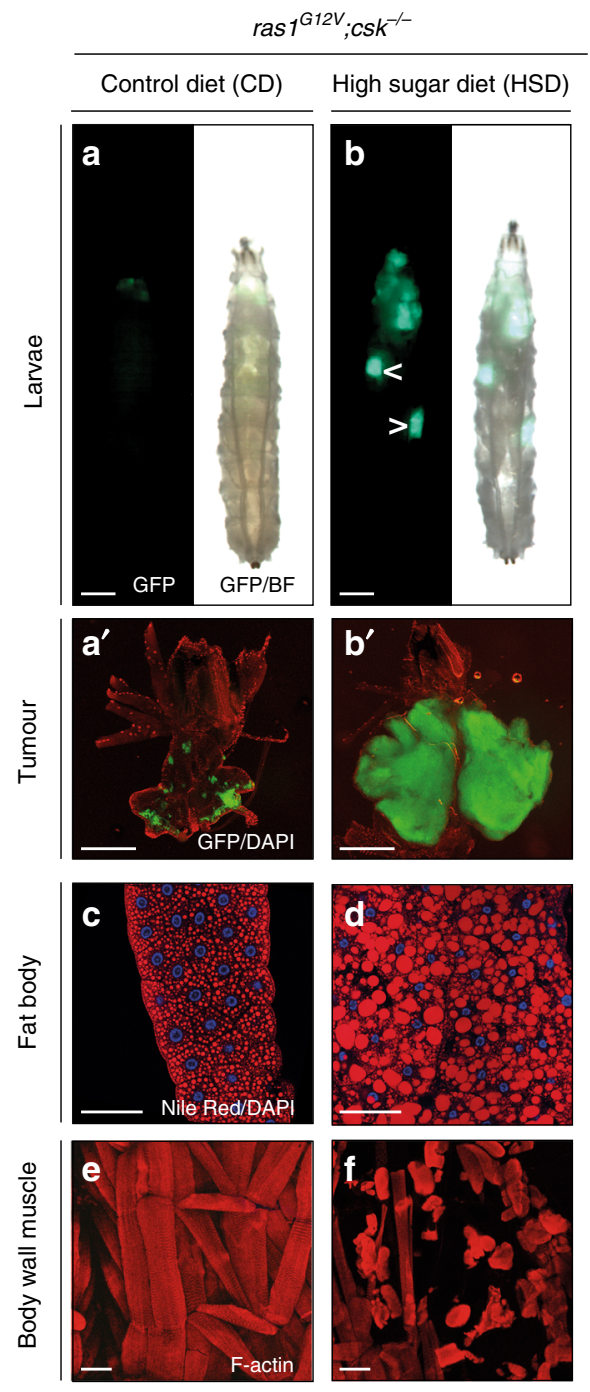

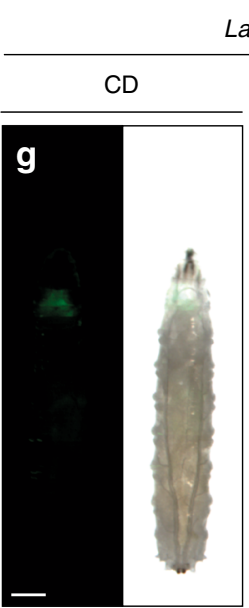

LacZ
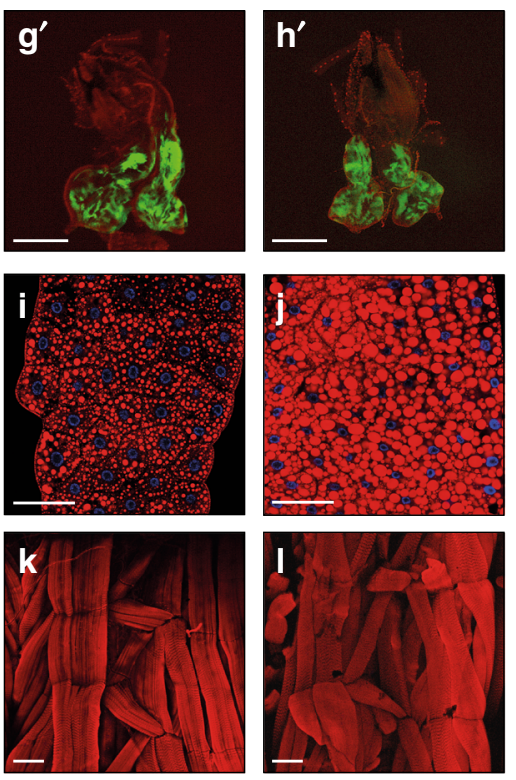

m Muscle wasting quantification

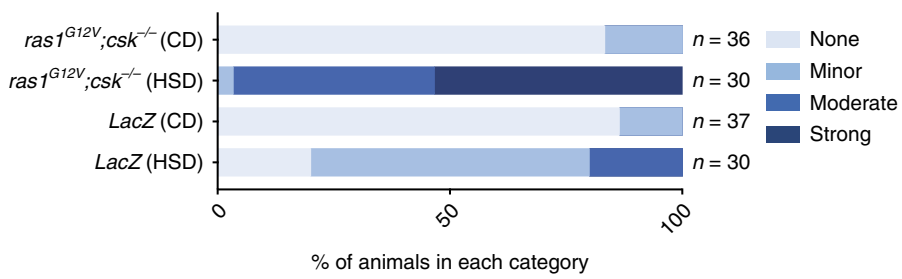

Fig. 1 HSD-fed Ras/Src-animals exhibit systemic muscle wasting. ras $7^{G 12 V}$; $c s k^{-/-}$third-instar larvae raised on CD (a) or HSD (b). Transformed tissue is labelled with GFP (green). Secondary tumours are observed in a subset of animals (arrowheads in $\mathbf{b}$ ). Scale bar, $500 \mu \mathrm{m}$. $\mathbf{a}^{\prime}, \mathbf{b}^{\prime}$ Matching dissected eye epithelial tissue stained with DAPI (red). Scale bar, $250 \mu \mathrm{m}$. Nile Red (red) and DAPI (blue) staining of dissected fat body tissue from ras ${ }^{G 12 V} ; \mathrm{csk}^{-/}{ }^{-}$thirdinstar larvae fed a CD (c) or HSD (d). Scale bar, $100 \mu \mathrm{m}$. F-actin (red) staining of dissected larval body-wall muscle tissue from ras 1 G12V; ;sk $^{-/ /}$- third-instar larvae raised on CD $(\mathbf{e})$ or HSD (f). Scale bar, $100 \mu \mathrm{m}$. LacZ third-instar larvae raised on CD (g) or HSD (h). LacZ-expressing eye tissue is labelled with GFP (green). Scale bar, $500 \mu \mathrm{m} . \mathbf{g}^{\prime}, \mathbf{h}^{\prime}$ Matching dissected eye epithelial tissue stained with DAPI (red). Scale bar, $250 \mu \mathrm{m}$. Nile Red (red) and DAPI (blue) staining of dissected fat body tissue from LacZ third-instar larvae fed a CD (i) or HSD (j). Scale bar, $100 \mu$ m. F-actin (red) staining of dissected larval bodywall muscle tissue from ras $1 G^{G} 2 V_{;}$csk ${ }^{-/-}$third-instar larvae fed a CD (k) or HSD (I). Scale bar, $100 \mu \mathrm{m}$. m Matching body-wall muscle wasting quantification. $X$-axis represents the percentage of individual animals scoring in each category denoted 'none', 'minor', 'moderate' or 'strong'.

tumour-derived factor that contributes to muscle wasting in Ras/ Src-animals in HSD.

Importantly, reducing $b n l$ expression in HSD-enhanced Ras/ Src-tumours (ras1 $\left.1^{G 12 V} ; c s k^{-/-}, b n l^{R N A i}\right)$ not only attenuated systemic muscle wasting but also suppressed tumour growth and larval lethality (Fig. $2 \mathrm{~d}-\mathrm{f}$ and Supplementary Fig. 3p). Tumour autonomous $\mathrm{Bnl}$ and its receptor Breathless (Btl) signalling has been implicated in tumourigenesis ${ }^{21}$. However, reducing the levels of $b t l$ in Ras/Src-activated tumours $\left(\right.$ ras $\left.1^{G 12 V}, b t l^{R N A i} ; c s k^{-/}\right)$had minimal effect on primary tumour size or muscle wasting, indicating that tumour autonomous reduction of Bnl-Btl signalling was not responsible for the suppression of tumour growth in $\operatorname{rasl}^{G 12 V_{;}}$csk ${ }^{-1}$, , bnl $l^{R N A i}$ animals (Supplementary Fig. $3 \mathrm{n}-\mathrm{q})$. Together, these results suggest that 
a Tumour-derived FGF ligands

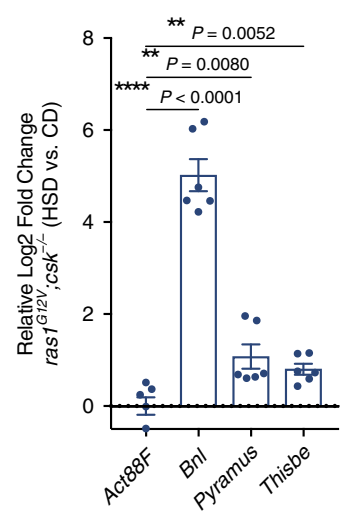

$\operatorname{ras} 1^{G 12 V} ; \mathrm{Csk}^{-1}$
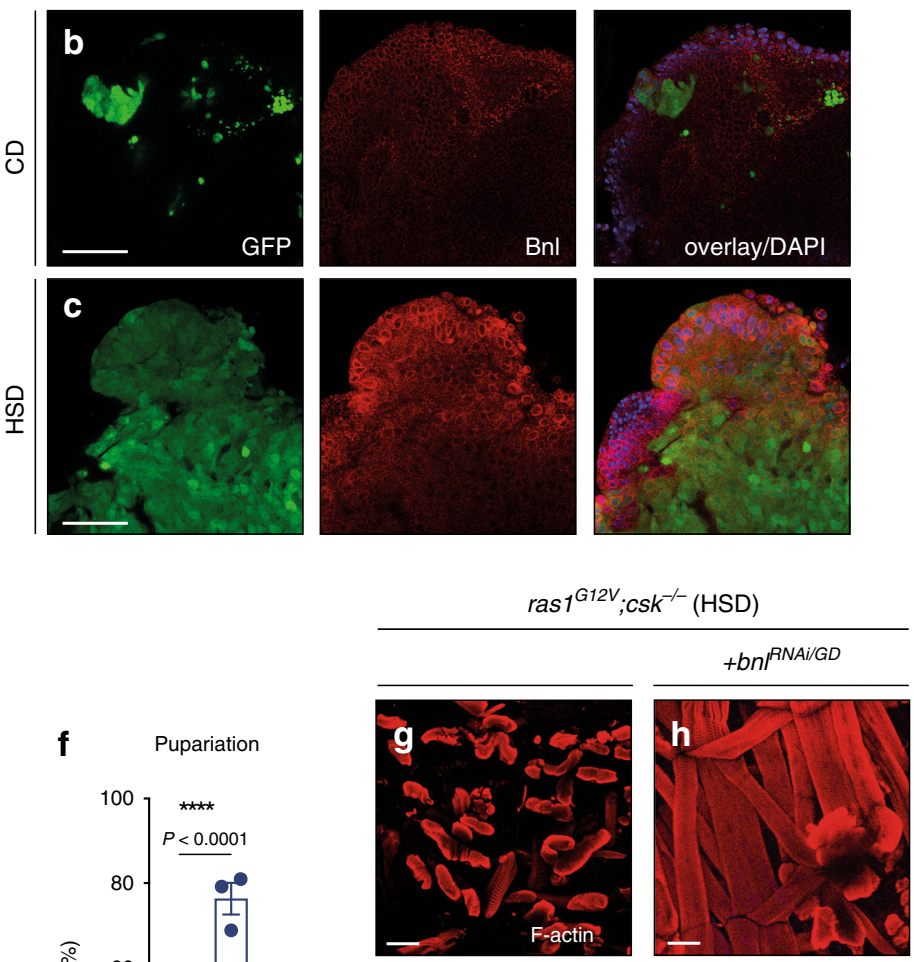

HSD

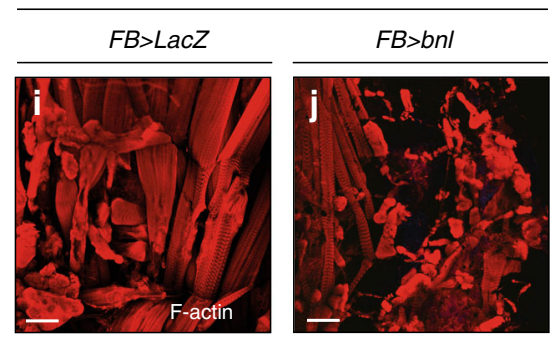

K Muscle wasting quantification

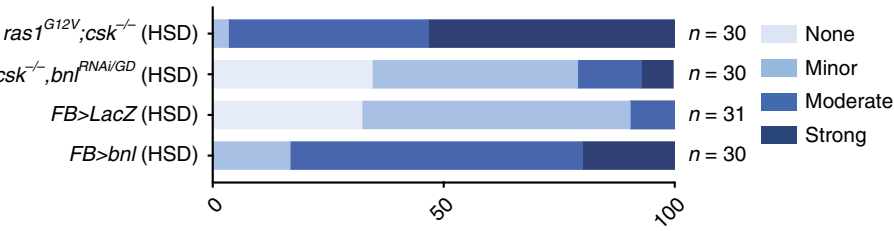

$\%$ of animals in each category

Fig. 2 Tumour-derived branchless mediates muscle wasting and tumour growth. a Relative log2fold change of Drosophila fibroblast growth factors, branchless $(b n l)$, pyramus and thisbe in dissected tumour tissue from ras $7^{G 12} V_{;} c s k^{-/-}$animals raised on HSD compared to animals raised on CD, as determined by qPCR. Samples are normalised to Act88F. Results are shown as mean \pm SEM. Data from $n=6$ biologically independent samples. Data were analysed by two-tailed unpaired Student's $t$ test. Asterisks indicate statistically significant difference $\left({ }^{\star \star} P<0.01 ;{ }^{\star \star \star \star} P<0.0001\right)$. Anti-Bnl staining (red) of dissected tumour tissue from ras $1 \mathrm{G}^{12 \mathrm{~V}} ; \mathrm{csk}^{-/-}$animals raised on CD (b) or HSD (c) with DAPI (blue). Scale bar, $40 \mu \mathrm{m}$. Third-instar larvae

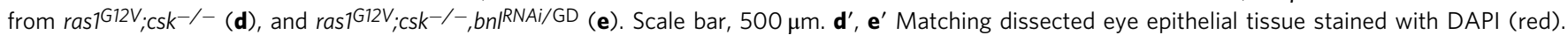
Scale bar, $250 \mu \mathrm{m}$. f Pupariation percentage of ras ${ }^{G 12 \mathrm{~V}} ; \mathrm{csk}^{-/-}$, and ras $1^{\mathrm{G} 12 \mathrm{~V}} ; \mathrm{csk}^{-/-}$, bn/RNAi/GD animals raised on HSD. Results are shown as mean $\pm \mathrm{SEM}$.

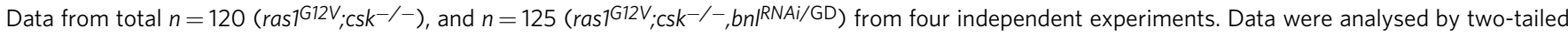
unpaired Student's $t$ test. Asterisks indicate statistically significant difference ( $\left.{ }^{\star \star \star \star} P<0.0001\right)$. F-actin (red) staining of dissected larval body-wall muscle

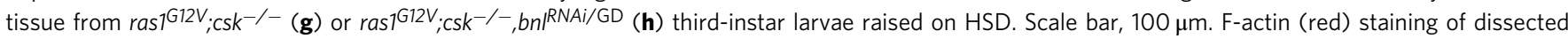
larval body-wall muscle tissue from $F B>L a c Z(\mathbf{i})$ or $F B>b n l(\mathbf{j})$ third-instar larvae raised on HSD. Scale bar, $100 \mu \mathrm{m}$. $\mathbf{k}$ Matching body-wall muscle wasting quantification. 
a Circulating amino acids
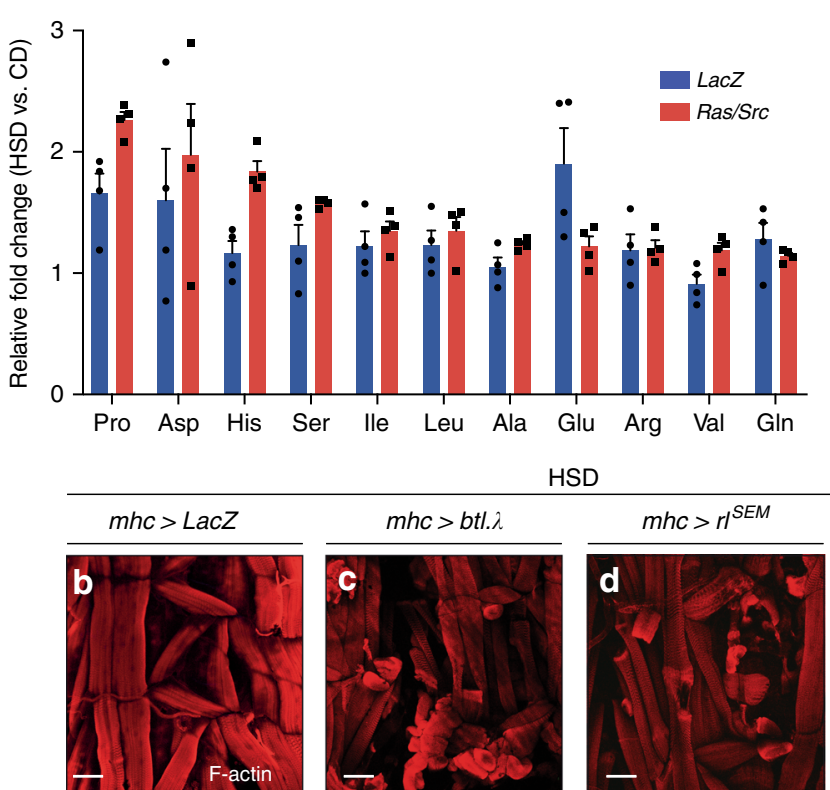

$m h c>b t l . \lambda$

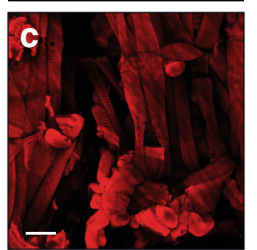
$m h c>r I^{S E M}$

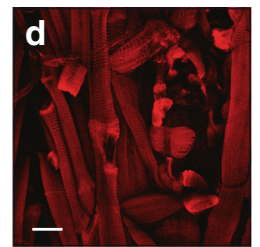

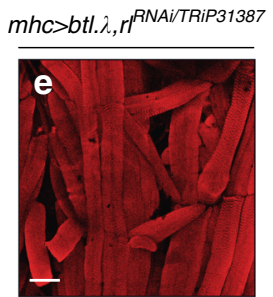

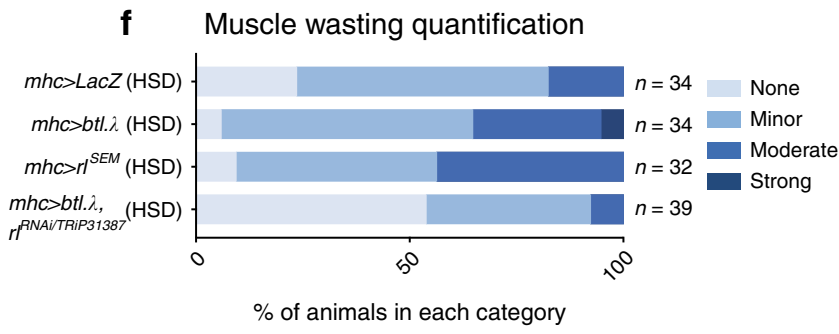

g

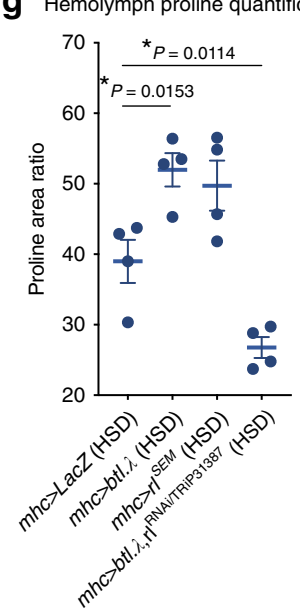

Fig. 3 Muscle-specific BtI-dERK signalling promotes muscle wasting in HSD. a Circulating haemolymph levels of upregulated amino acids in LacZ (blue bars) and ras ${ }^{G 12 V}$; csk $^{-/-}$(red bars) animals raised on HSD compared to animals raised on CD. Results are shown as mean \pm SEM. Data from $n=4$ biologically independent samples. F-actin (red) staining of dissected larval body-wall muscle tissue from $m h c>L a c Z(\mathbf{b}), m h c>b t l . \lambda(\mathbf{c}), m h c>r \mid S E M(\mathbf{d}), a n d$ $m h c>b t l . \lambda, r l^{R N A i / T R i P 31387}$ (e) third-instar larvae raised on HSD. Scale bar, $100 \mu \mathrm{m}$. f Matching body-wall muscle wasting quantification. $\mathbf{g}$ Matching haemolymph proline quantification. Results are shown as mean \pm SEM. Data from $n=4$ biologically independent samples. Data were analysed by twotailed unpaired Student's $t$ test. Asterisks indicate statistically significant difference $\left({ }^{\star} P<0.05\right)$.

systemic muscle wasting has a functional effect on tumour growth.

Muscle wasting associates with circulating proline. A consequence of skeletal muscle wasting is protein degradation and subsequent release of free amino acids into the circulation. Through targeted metabolomic analysis, we measured an increase in circulating levels of amino acids in the haemolymph of tumour-free LacZ-animals raised on an HSD relative to CD (Fig. 3a and Supplementary Fig. 4a). Consistent with enhanced muscle wasting in the presence of tumours (Fig. 1f, l, m), circulating amino acids were further elevated in $\mathrm{rasl}^{\mathrm{G} 12 \mathrm{~V}} ; \mathrm{csk} \mathrm{K}^{-/-}$animals raised on an HSD (Fig. 3a and Supplementary Fig. 4a). Together with increased circulating 3-methylhistidine, a biomarker for muscle atrophy 22 (Supplementary Fig. 4b), these results indicate that in tumour-bearing animals raised on an HSD, circulating levels of amino acids are further elevated.

Ectopic expression of $b n l$ from the fat body not only promoted muscle wasting in HSD (Fig. 2i-k), but also led to elevated proline -the most elevated amino acid in the circulation-in the haemolymph (Supplementary Fig. 4c). Furthermore, reducing $b n l$ expression in HSD-enhanced Ras/Src-tumours (ras $1^{G 12 V}$; $c s k^{-/-}, b n l^{R N A i}$ ) not only attenuated systemic muscle wasting (Fig. 2g, h and Supplementary Fig. 3f, g, p), but also reduced circulating levels of proline (Supplementary Fig. 4d). These results support the association of ectopic- or tumour-derived Bnl with muscle wasting and circulating proline levels.

We performed a genome-wide transcriptional profiling analysis of dissected body-wall muscle from $\mathrm{ras}^{\mathrm{G12V}} ; \mathrm{csk} \mathrm{K}^{-/-}$animals raised on CD and HSD (Supplementary Fig. 4e). Gene set enrichment analysis (GSEA) revealed a progressive deregulation of muscle structure organisation in $\operatorname{ras} 1^{G 12 V} ; c s k^{-/-}$animals in HSD (Supplementary Fig. 4f). Bnl binds to its only receptor breathless $(b t l)$ and activates downstream Ras-ERK signalling ${ }^{23}$. Elevated ERK signalling promotes muscle wasting in adult Drosophila and in C. elegans ${ }^{11,24}$. GSEA analysis of muscle RNA-sequencing data revealed an increase in ERK-signalling pathway activity in $\operatorname{rasl}^{G 12 V} ; c s k^{-/-}$animals in HSD, which correlated with increased phospho-ERK (pERK) staining (Supplementary Fig. 4g-i). Muscle-specific expression of an active form of btl $(b t l . \lambda)$ or ERK $\left(r l^{S E M}\right)$ promoted muscle wasting and circulating proline levels (Fig. $3 \mathrm{~b}-\mathrm{d}, \mathrm{f}, \mathrm{g}$ ). Reducing the expression of ERK in this setting $\left(m h c>b t l . \lambda, r l^{R N A i}\right)$ rescued muscle wasting and proline levels (Fig. $3 \mathrm{e}-\mathrm{g}$ and Supplementary Fig. $4 \mathrm{j}-\mathrm{l}$ ). Overall, these analyses demonstrate that muscle-specific activation of btl-ERK signalling promotes muscle wasting which correlates with circulating proline levels. Although our data suggest that tumour-derived bnl may promote muscle wasting through btl in the muscle, tumour-derived bnl may also mediate muscle wasting indirectly through other mechanisms.

SLC36-family transporters are required for tumour growth. Since amino acids are one of the largest contributors to increased cell mass in proliferating cells ${ }^{1}$, our observations suggested muscle wasting as a source of circulating amino acids for tumour progression. To explore candidates that could be responsible for tumour-specific amino acid utilisation, we focused on amino acid transporters. Through RNA-sequencing analysis of tumour tissue from ras $1^{G 12 V} ; c^{-} k^{-}-$animals raised on CD and HSD, we characterised the expression profile of amino acid transporters across 
the SLC1, SLC7, SLC36 and SLC38 families (Supplementary Fig. 5a). Subsequent qPCR validation confirmed that amino acid transporters of the SLC7 family (minidiscs (mnd) and JhI-21) and SLC36 family (CG8785, pathetic (path) and CG1139) were upregulated in tumours of ras $^{G 12 V} ;$ csk ${ }^{-/-}$animals raised on an HSD compared to CD (Fig. 4a). Of note, expression levels of Slimfast (slif)-another SLC7 family member and an amino acid transporter previously implicated in tumour-specific usage of amino acids $^{12,25}$-were downregulated in Ras/Src-tumours in HSD (Fig. 4a).

Reducing the expression of $m n d\left(r a s 1^{G 12 V} ; c s k^{-/-}, m n d^{R N A i}\right.$ ),

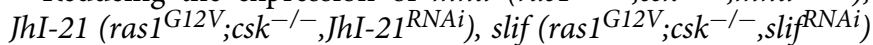
or CG8785 (ras1 ${ }^{G 12 V} ;$ csk $\left.^{-/-}, C G 8785^{R N A i}\right)$ had no effect on primary tumour size (Fig. 4b-f and Supplementary Fig. 5g). Strikingly, reducing expression of path ( $\mathrm{ras}^{\mathrm{G} 12 \mathrm{~V}}$,path ${ }^{R N A i}$; $c^{-1-}$ ) or CG1139 (ras1 ${ }^{G 12 V}$; $c k^{-/-}, C G 1139^{R N A i}$ ) almost completely suppressed HSD-mediated Ras/Src-tumour growth (Fig. 4g, h and Supplementary Fig. 5b, c, g). Importantly, reduction in path or CG1139 levels had minimal effect on tumour growth in ras $1^{G 12 V} ; c s k^{-1-}$ animals fed a CD (Supplementary Fig. $5 \mathrm{~d}-\mathrm{g}$ ), indicating that neither is required for benign tumour growth. Altogether, these results demonstrate that Path and CG1139 are specifically required for HSD-mediated enhancement of Ras/Src-tumour growth.

We confirmed elevation of Path and CG1139 protein levels in Ras/Src-tumours in HSD using the Path- and CG1139-specific antibodies $^{26}$ (Fig. 4i, j, m, n and Supplementary Fig. 5h-k). Path has been previously identified as one of the growth-regulatory factors under the control of the Hippo signalling pathway downstream transcriptional co-activator Yorkie (Yki) ${ }^{27}$. Overexpression of Warts (Wts) - and therefore inhibition of Ykiactivity-in Ras/Src-activated cells ( $\mathrm{ras}^{\mathrm{G} 12 V} ; \mathrm{csk}^{-/-}$, wts) led to decreased Path protein but not CG1139 levels in animals fed an HSD (Fig. 4k, o). Conversely, the expression of Yki in Ras/Srcactivated cells $\left(\mathrm{ras}^{\mathrm{G12}} ; \mathrm{csk}^{-/-}, \mathrm{Yki}\right)$ led to increased path transcript and Path protein expression in animals raised on $\mathrm{CD}$, but not CG1139 (Fig. 4l, p and Supplementary Fig. 5j-1). While Path was elevated specifically in Ras/Src-activated tumours in HSD (Fig. 4j, $\mathrm{k}$ and Supplementary Fig. 5h), the elevation of CG1139 was not specific to tumours, but was rather dependent on HSD (Fig. 4n, o and Supplementary Fig. 5i). Together with our previous study demonstrating that HSD-enhanced Ras/Src-tumours activate $\mathrm{Yki}^{14}$, we conclude that the Hippo signalling downstream effector Yki mediates path expression in ras $^{G 12 V} ; c s k^{-/-}$animals raised on an HSD.

Proline promotes tumour growth via Path-Tor-S6K signalling. Mammalian SLC36-family amino acid transporters including SLC36A1 and SLC36A2 transport alanine, glycine and proline ${ }^{28}$. Proline was the most highly increased amino acid in the haemolymph of ras1 $1^{G 12 V} ; c s k^{-/-}$animals in HSD (Fig. 3a and Supplementary Fig. 4a). Overexpression of Path $\left(\operatorname{ras}^{G 12 V_{;}} ; \mathrm{cs}^{-/-}\right.$, path) or CG1139 (ras1 ${ }^{G 12 V}, C G 1139 ;$ csk $^{-/-}$) had a minor, yet insignificant effect on tumour growth in Ras/Src-activated cells on CD (Fig. 5a-c and Supplementary Fig. 6a).

To mimic the elevation of circulating proline levels under conditions of muscle wasting, we carried out dietary supplementation of proline. Feeding animals CD supplemented with 100 $\mathrm{mM} \mathrm{L}$-proline had minimal effect on tumour growth of $\operatorname{ras}^{\mathrm{G12}} \mathrm{V}$; $c s k^{-1-}$ animals in CD (Fig. $5 \mathrm{~d}$ and Supplementary Fig. 6a). Intriguingly, however, feeding proline in $\mathrm{ras}^{\mathrm{G12V}} ; \mathrm{csk}^{-\rho_{-}}$, path animals strongly promoted tumour growth in $\mathrm{CD}$ (Fig. 5e and Supplementary Fig. 6a). In striking contrast, proline feeding in ras1 ${ }^{G 12 V}, C G 1139 ; c s k^{-/-}$animals had no effect on tumour growth (Fig. $5 \mathrm{f}$ and Supplementary Fig. 6a). Of note, feeding proline in animals with path or CG1139 overexpressing clones had a negligible effect on eye tissue growth (Fig. 5g-i and Supplementary Fig. 6b), suggesting that proline-Path-mediated enhancement of tissue growth requires an oncogenic background. Feeding the biologically inert isomer, D-proline had no tumour-promoting effect in both ras1 $1^{G 12 V} ; c s k^{-/-}$and $\mathrm{ras}^{\mathrm{G12V}} ; \mathrm{csk}^{-/-}$, path animals (Supplementary Fig. 6e, h, k), thereby indicating that tumour response is specific to $\mathrm{L}$-proline. Feeding animals $\mathrm{CD}$ supplemented with $100 \mathrm{mM}$ of stable isotope of L-proline $\left({ }^{13} \mathrm{C}^{15} \mathrm{~N}\right.$ proline) led to increased proline in $\mathrm{ras}^{\mathrm{G12V}} ; \mathrm{csk} \mathrm{K}^{-/}$, path tumours but not in $\operatorname{ras}^{G 12 V}, C G 1139 ;$ csk $^{-/-}$tumours (Fig. 5j), revealing that Path mediates tumour uptake of circulating proline. While feeding excess L-glycine marginally promoted growth of rasi $1^{G 12 V_{;}}$csk ${ }^{-/-}$,path tumours, L-alanine had no growthpromoting effect in $\operatorname{ras} 1^{G 12 V} ; c s k^{-/-}, \operatorname{ras} 1^{G 12 V} ; c s k^{-/-}$,path or ras1 ${ }^{G 12 V}, C G 1139 ;$ csk $^{-/-}$animals (Supplementary Fig. 6c, d, f, g, i-k). Altogether, our data highlight the proline vulnerability of HSD-enhanced Ras/Src-tumours and uncover modulation of amino acid transporter repertoire as a strategy to meet the nutrient requirements of tumourigenesis.

Tor-S6K signalling is implicated downstream of SLC36 transporters ${ }^{29,30}$. Expression of a dominant-negative isoform of Tor in Ras/Src-activated cells ( $\left.\operatorname{ras}^{G 12 V}, \operatorname{Tor}^{T E D} ; \mathrm{csk}^{-/-}\right)$suppressed tumour growth in HSD (Fig. 5k, 1 and Supplementary Fig. 61). Conversely, expression of an active isoform S6K in Ras/ Src-activated cells (ras1 $1^{G 12 V} ; c s k^{-/}$,S6KSTDETE) promoted tumour growth in CD (Fig. 5m, n and Supplementary Fig. 61). Increased phospho-specific S6 (pS6) antibody ${ }^{31}$ staining was observed in rasi $1^{G 12}$; $c s k^{-/-}$tumours of animals in HSD compared to $\mathrm{CD}$ (Fig. 5o, p, s). Consistently, increased phospho-S6 staining was observed autonomously within ras1 $1^{G 12 V_{;}}$csk $k^{-/-}$,path tumours but not in $\operatorname{ras}^{\mathrm{Gl}}{ }^{2 \mathrm{~V}}, \mathrm{Tor}^{\mathrm{TED}}$ $c s k^{-/}$,path tumours of animals raised on a proline-supplemented diet (Fig. $5 q-s$ ). These results demonstrate that proline-Path promotes Tor-S6K signalling.

We next examined the link between Path-mediated Ras/Srctumour growth and muscle wasting. Feeding proline in $\operatorname{rasl}^{\mathrm{G1}} \mathrm{V}$; $c s k^{-/-}$,path animals not only promoted tumour growth (Fig. 5e), but also led to elevated tumour expression of Bnl (Supplementary Fig. $7 \mathrm{a}, \mathrm{b}, \mathrm{h}$ ) and muscle wasting in animals raised on CD (Supplementary Fig. 7c-e). In addition, $\operatorname{ras} 1^{\mathrm{G} 12 V_{;}} \mathrm{csk} \mathrm{K}^{-/}$, $S 6 K^{S T D E T E}$ tumours promoted expression of Bnl, suggesting that increased Path-Tor-S6K signalling promotes bnl expression (Supplementary Fig. $7 \mathrm{f}-\mathrm{h}$ ). Together these results demonstrate that proline-Path-mediated tumour growth promotes Bnl expression and muscle wasting. Overall, our data implicate the coordinate induction of tumour-autonomous amino acid transporter expression, coupled with systemic muscle wasting, as sufficient to establish a feed-forward host-tumour circuit to drive tumour growth.

Targeting proline vulnerability of tumours. Our work highlights SLC36-family transporters as a target for therapeutic intervention in tumours with proline vulnerability. A previous in vitro study using a human cancer cell line explored amino acid and amino acid derivative specificities of SLC36A1 ${ }^{32}$. By selecting competitive, non-transported inhibitors of SLC36A1 from this study, we performed a whole-animal Drosophila screen to identify candidates that suppress tumour growth and display minimal whole-animal toxicity. Feeding $\operatorname{ras} 1^{G 12 V_{;}}$csk ${ }^{-/-}$animals an HSD supplemented with 5-Hydroxy-L-tryptophan led to animal lethality at the early larval stages, indicative of whole-animal toxicity. Feeding ras $1^{G 12 V_{;}} ; s k^{-/-}$animals an HSD supplemented with either L-tryptophan or Indole-5-carboxylic acid led to successful larval development, but had minimal inhibitory effect on tumour 
a Amino acid transporter expression

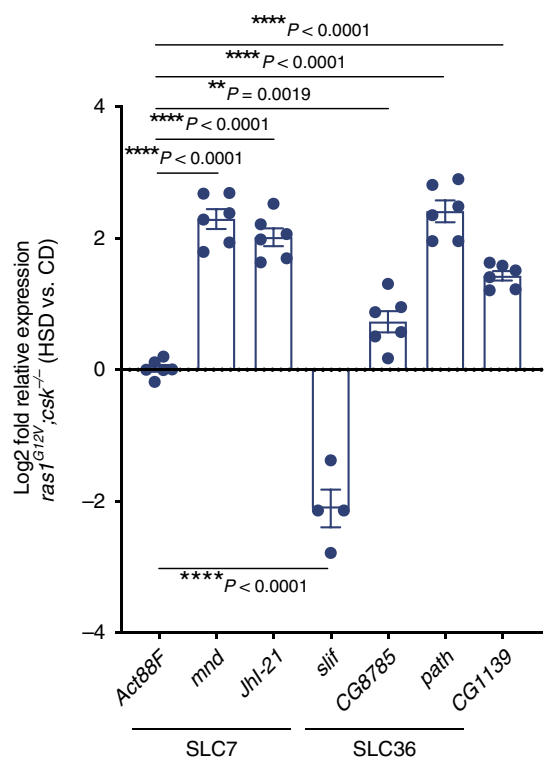

$\operatorname{ras} 1^{\mathrm{G} 12 \mathrm{~V}} ; \mathrm{csk}^{-/-}$(HSD)
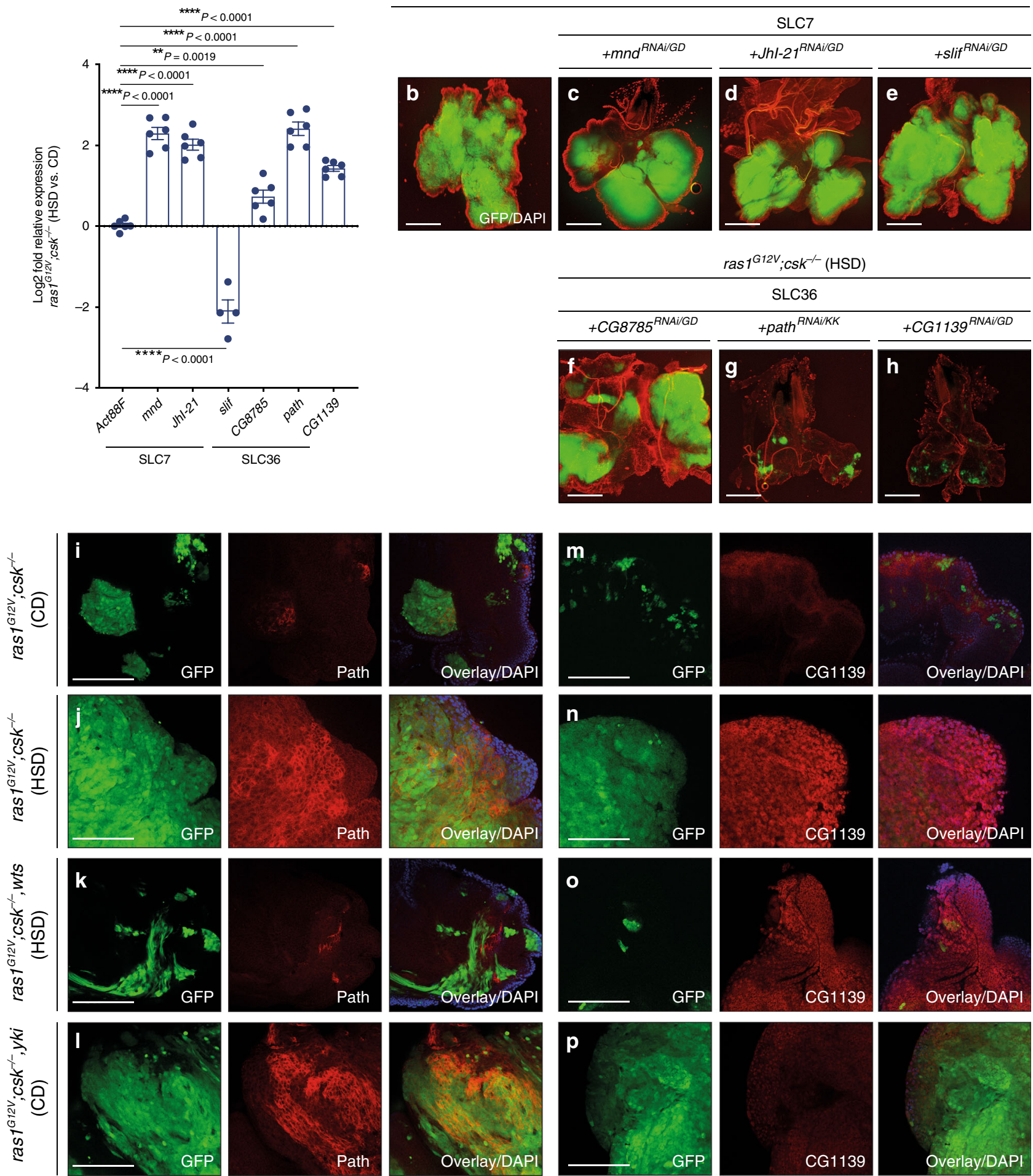

Fig. 4 Amino acid transporter Path is required for HSD-enhanced Ras/Src-tumour growth. a Relative log2fold change of SLC7- and SLC36-family amino acid transporters in dissected tumour tissue from ras ${ }^{G 12 V_{i}} ; \mathrm{csk}^{-/-}$animals raised on HSD compared to animals raised on CD, as determined by $\mathrm{qPCR}$. Samples are normalised to Act88F. Results are shown as mean \pm SEM. Data from $n=6$ (Act88F), $n=6(\mathrm{mnd}), n=6$ (Jhl-21), $n=4$ (slif), $n=6($ CG8785), $n=6$ (path) and $n=6$ (CG1139) biologically independent samples. Data were analysed by two-tailed unpaired Student's $t$ test. Asterisks indicate statistically significant difference ( $\left.{ }^{\star \star} P<0.01 ;{ }^{\star \star \star \star} P<0.0001\right)$. Dissected eye epithelial tissue stained with DAPI (red) from ras ${ }^{G 12 V ; \mathrm{csk}{ }^{-/}-}$animals raised on $\operatorname{HSD}(\mathbf{b})$, with mndRNAi/GD (c), Jhl-27RNAi/GD (d), slifRAi/GD (e), CG8785RNAi/GD (f), path ${ }^{R N A i / K K}$ (g) and CG1139RNAi/GD (h). Scale bar, 250 $\mu$ m. Anti-Path

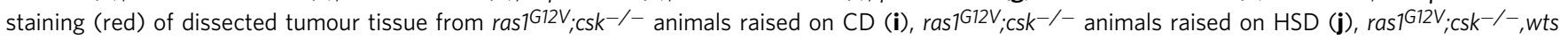
animals raised on $\mathrm{HSD}(\mathbf{k})$ and ras $1^{G 12 V_{;}} ; \mathrm{csk}^{-/-}, y \mathrm{ki}$ animals raised on CD (I) with DAPI (blue). Scale bar, $100 \mu \mathrm{m}$. Anti-CG1139 staining (red) of dissected

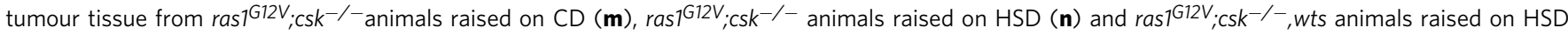
(o), and ras ${ }^{G 12 V} ; c^{c s k}{ }^{-/-}, y k i$ animals raised on CD (p) with DAPI (blue). Scale bar, $100 \mu \mathrm{m}$. 

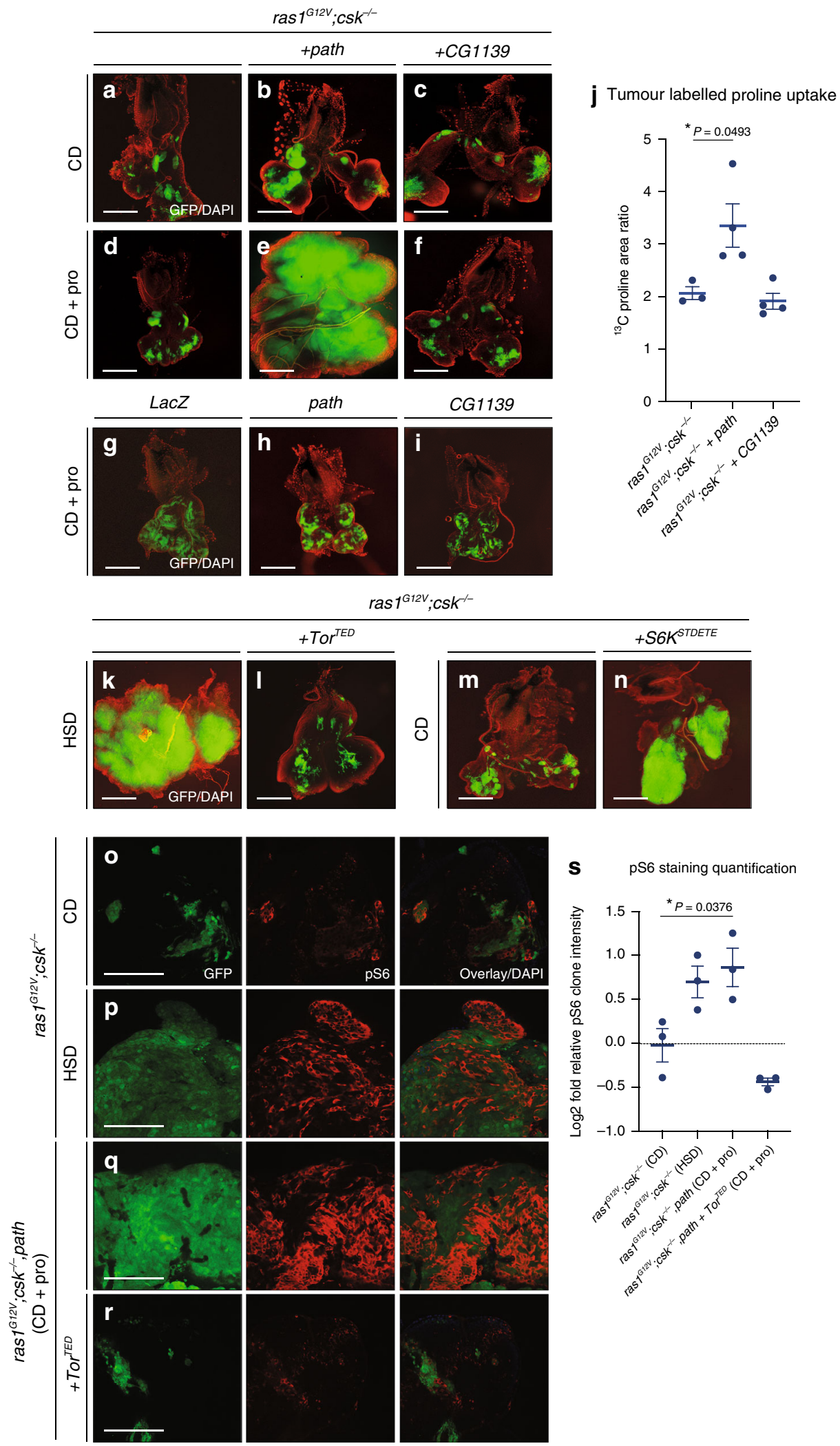

growth (Fig. 6a-c and Supplementary Fig. 8a). However, feeding ras $1^{G 12 V} ; c s k^{-/-}$animals an HSD supplemented with Indole-3propionic acid (IPA) dramatically suppressed tumour growth in a dose-dependent manner (Fig. 6d and Supplementary Fig. 8a-f). Strikingly, $66.2 \%$ of IPA-fed $\mathrm{ras}^{\mathrm{G12V}} ; \mathrm{csk} \mathrm{K}^{-/-}$animals achieved pupariation in HSD, revealing significant tumour-suppressing efficacy coupled with minimal whole-animal toxicity (Fig. 6g-i and Supplementary Fig. 8g). IPA feeding had no effect on benign tumour growth in ras $1^{G 12 V} ; c s k^{-/-}$animals raised on CD (Fig. 6e, $\mathrm{f}$ and Supplementary Fig. $8 \mathrm{~h}$ ) supporting the notion that SLC36 inhibition only limits the growth of tumours with proline vulnerability. Furthermore, IPA suppressed $\operatorname{ras1}^{\mathrm{G} 12 V_{;}} ; \mathrm{csk}^{-/-}$,pathtumour growth in proline-fed animals in $\mathrm{CD}$, and increasing the dietary concentrations of proline partially out-competed IPA- 
Fig. 5 Proline promotes Ras/Src-tumour growth through amino acid transporter Path. Dissected eye epithelial tissue stained with DAPI (red) from

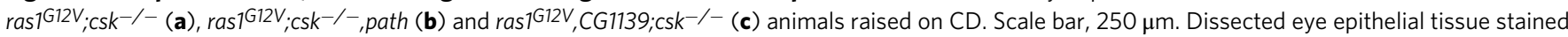

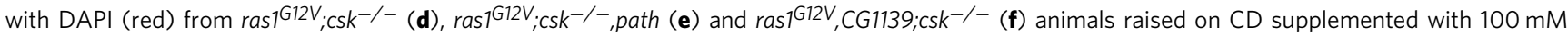
L-proline. Scale bar, $250 \mu \mathrm{m}$. Dissected eye epithelial tissue stained with DAPI (red) from LacZ (g), Path (h) and CG1139 (i) animals raised on CD

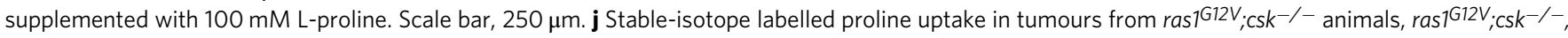
path animals and ras1G12V, CG1139; $\mathrm{csk}^{-/-}$animals raised on CD supplemented with $100 \mathrm{mM}{ }^{13} \mathrm{C}$-labelled L-proline. Results are shown as mean $\pm \mathrm{SEM}$. Data from $n=3\left(\right.$ ras $\left.^{G 12 V} ; \mathrm{csk}^{-/-}\right), n=4\left(\mathrm{ras}^{\mathrm{G} 12 \mathrm{~V}} ; \mathrm{csk}^{-/-}\right.$, path) and $n=4\left(\mathrm{ras}^{\mathrm{G} 12 \mathrm{~V}}, \mathrm{CG} 1139 ; \mathrm{csk}^{-/-}\right)$biologically independent samples. Data were analysed by two-tailed unpaired Student's $t$ test. Asterisks indicate statistically significant difference $\left({ }^{\star} P<0.05\right)$. Dissected eye epithelial tissue stained with DAPI

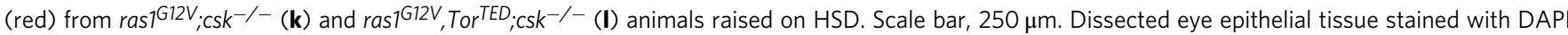
(red) from ras7 ${ }^{G 12 V_{;}}$csk $^{-/-}(\mathbf{m})$ and ras7G12V; S6KSTDETE, csk $^{-/-}(\mathbf{n})$ animals raised on CD. Scale bar, $250 \mu$ m. Anti-phospho-S6 (pS6) staining (red) of

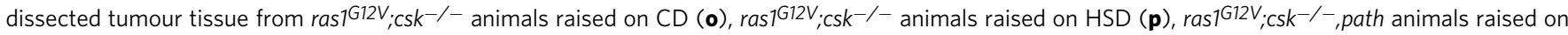

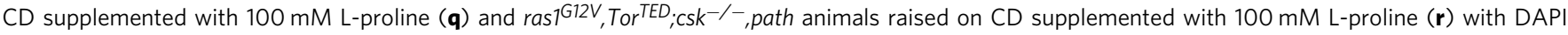
(blue). Scale bar, $100 \mu \mathrm{m}$. s Matching phospho-S6 (pS6) staining quantification. Results are shown as mean \pm SEM. Data from $n=3$ biologically independent samples. Data were analysed by two-tailed unpaired Student's $t$ test. Asterisks indicate statistically significant difference $\left({ }^{\star} P<0.05\right)$.

inhibition (Fig. 6j-1 and Supplementary Fig. 8i). These data indicate that IPA specifically targets the proline dependency of tumour growth.

A previous report identified exogenous proline dependent cell lines, including pancreatic cancer cell line Panc $04.03^{3}$. The cell culture media DMEM lacks proline and other non-essential amino acids (NEAAs), whereas RPMI includes all NEAAs. Panc.04.03 cells exhibited increased proliferation in RPMI compared to DMEM (Supplementary Fig. 8j). Supplementation of proline into DMEM promoted cell proliferation, indicating that these cell lines are responsive to exogenous proline (Supplementary Fig. 8j). Furthermore, addition of IPA in the culture medium attenuated cell proliferation in prolinesupplemented DMEM (Supplementary Fig. 81, m). Although IPA had a minor effect on cell proliferation in DMEM, IPA suppresses enhanced cell proliferation which is mediated by exogenous proline (Supplementary Fig. 8k, m).

\section{Discussion}

Muscle wasting is observed in chronic muscle wasting diseases due to cancer (cachexia), ageing/senescence (sarcopenia), myopathies and other metabolic diseases, as well as in acute conditions due to burns and sepsis ${ }^{33}$. In this study, we demonstrate that the combination of diet-induced obesity and tumour growth induces systemic muscle wasting (Fig. 1), associated with functional locomotion defect (Supplementary Fig. 1o, p). This muscle phenotype was not due to the inability to properly form muscle during development; the muscles are wholly formed at the early stage of larval development, and waste progressively at the later larval stage, as the tumours develop (Supplementary Fig. 1k-m). In addition, we demonstrate that the muscle phenotype observed in our model is unrelated to a developmentally related degeneration process (Supplementary Fig. 2).

Our study reveals a systemic amino acid-utilising circuit whereby HSD-enhanced tumours induce muscle wasting as a systemic metabolic network to drive tumourigenesis. A consequence of muscle wasting in $\operatorname{ras} 1^{G 12 V_{;}} ; s k^{-/-}$animals raised on HSD was increased release of proline into the circulation (Fig. 3a). Plasma amino acid profiling of patients with sarcopenia - a condition of muscle wasting associated with aging-showed elevated plasma proline levels ${ }^{34}$, indicating that elevated free circulating proline is a common feature of muscle wasting. We identify a proline vulnerability of HSD-enhanced tumours; SLC36-family amino acid transporter Path is required for tumour growth and exogenous proline promotes tumourigenesis through Path (Figs. 4 and 5). We highlight two layers of coordination in tumour metabolic response: (1) at the whole organism level-by promoting muscle wasting and systemic amino acid availability, and (2) at the tumour-autonomous level-by altering amino acid transporter repertoire (Fig. $6 \mathrm{~m}$ ).

Path and CG1139 have different transport characteristics for proline, with Path having a lower transport capacity compared to CG113930. Despite this, our labelled proline uptake experiments indicated uptake of extracellular proline in Ras/Src/Path-tumours but not in Ras/Src/CG1139-tumours (Fig. 5j), suggesting that CG1139 and Path may exhibit different activities in the context of an oncogenic background. Consistent with a previous report ${ }^{30}$, our data support the signalling role of Path through activation of the Tor-S6K pathway. Proline metabolism has been demonstrated to support cancer clonogenicity and metastasis 3,35 . Furthermore, proline promotes cancer cell survival under nutrient-limited or hypoxic microenvironments ${ }^{4,36}$. Our data extend these studies to reveal a tumour-promoting role of proline in response to systemic host metabolic changes.

IPA specifically targets the proline dependency of tumour growth in Drosophila (Fig. 6). Furthermore, we demonstrate a functional effect of IPA on human cells (Supplementary Fig. $8 j-m)$. A recent study demonstrated that proline uptake in Ras-driven tumour cells is much higher in spheroids in threedimensional cultures-which better mimic conditions in vivocompared to monolayers in two-dimensional cultures ${ }^{35}$, suggestive of a potentially stronger effect in tumours that are dependent on proline for growth in vivo. Proline is a limiting amino acid for protein synthesis in kidney cancers ${ }^{37}$. Therefore, reducing proline uptake with SLC36-inhibitors-as exemplified here by use of IPA (Fig. 6)-may warrant consideration as a therapeutic strategy to break the nutritional circuit between systemic muscle wasting and tumour growth in proline vulnerable cancers.

\section{Methods}

Fly stocks. UAS-lacZ (BDSC: 8529$)$, ecd ${ }^{1}$ (BDSC: 218$), U A S-b n l^{R N A i / T R i P}$ (BDSC 34572), UAS-ImpL2 $2^{\text {RNAi/TRiP }}$ (BDSC: 55855), UAS-Pvf2 $2^{\text {RNAi/TRiP }}$ (BDSC: 61955),

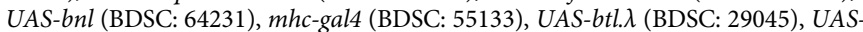
$r^{S E M}$ (BDSC: 59006), and UAS-rl ${ }^{\text {RNAi/TRiP }}$ (BDSC: 31387), UAS- $r l^{R N A i / T R i P}$ (BDSC: 31524), UAS-path ${ }^{\text {RNAi/TRiP (BDSC: 64029), UAS-wts (BDSC: 44258), UAS-Tor TED }}$ (BDSC: 7013), and UAS-S6KSTDETE (BDSC: 6914) flies were obtained from the Bloomington Drosophila Stock Centre.

UAS-bnl ${ }^{R N A i / G D}$ (VDRC: 5730), UAS-ImpL2 $2^{R N A i / G D}$ (VDRC: 30930), UAS$P v f 1^{R N A i / G D}$ (VDRC: 6173), UAS-Pvf1 ${ }^{R N A i / K K}$ (VDRC: 102699), UAS-Pvf2 ${ }^{R N A i / K K}$ (VDRC: 102072), UAS-btl ${ }^{R N A i / G D}$ (VDRC: 950), UAS-btl ${ }^{R N A i / K K}$ (VDRC: 110277), UAS-mnd ${ }^{R N A i / G D}$ (VDRC: 42485), UAS-slifRNAi/GD (VDRC: 45590), UAS-JhI$21^{R N A i / G D}$ (VDRC: 45193), UAS-CG8785 RNAi/GD (VDRC: 4650), UAS-path ${ }^{R N A i / K K}$ (VDRC: 100519), UAS-CG1139RNAi/GD (VDRC: 8907) and UAS-CG1139RNAi/KK (VDRC: 102363) flies were obtained from the Vienna Drosophila Resource Centre. The following stocks were kindly provided to us: FRT82B, csk ${ }^{Q 156 S t o p}$ by A. O'Reilly and M. Simon, ey(3.5)-FLP1 and UAS-ras1 $1^{G 12 V}$ by G. Halder, FB-gal4 by R. Kühnlein, UAS-pathA by J. Parrish, UAS-CG1139 by D. Goberdhan and UASYki.V5 by K. Irvine.

To create eyeless-driven GFP-labelled clones, the MARCM system was used. Flies with the genotype ey(3.5)-FLP1; act >y+ > gal4,UAS-GFP; FRT82B, tub-gal 80 were crossed to flies with the following genotypes: 

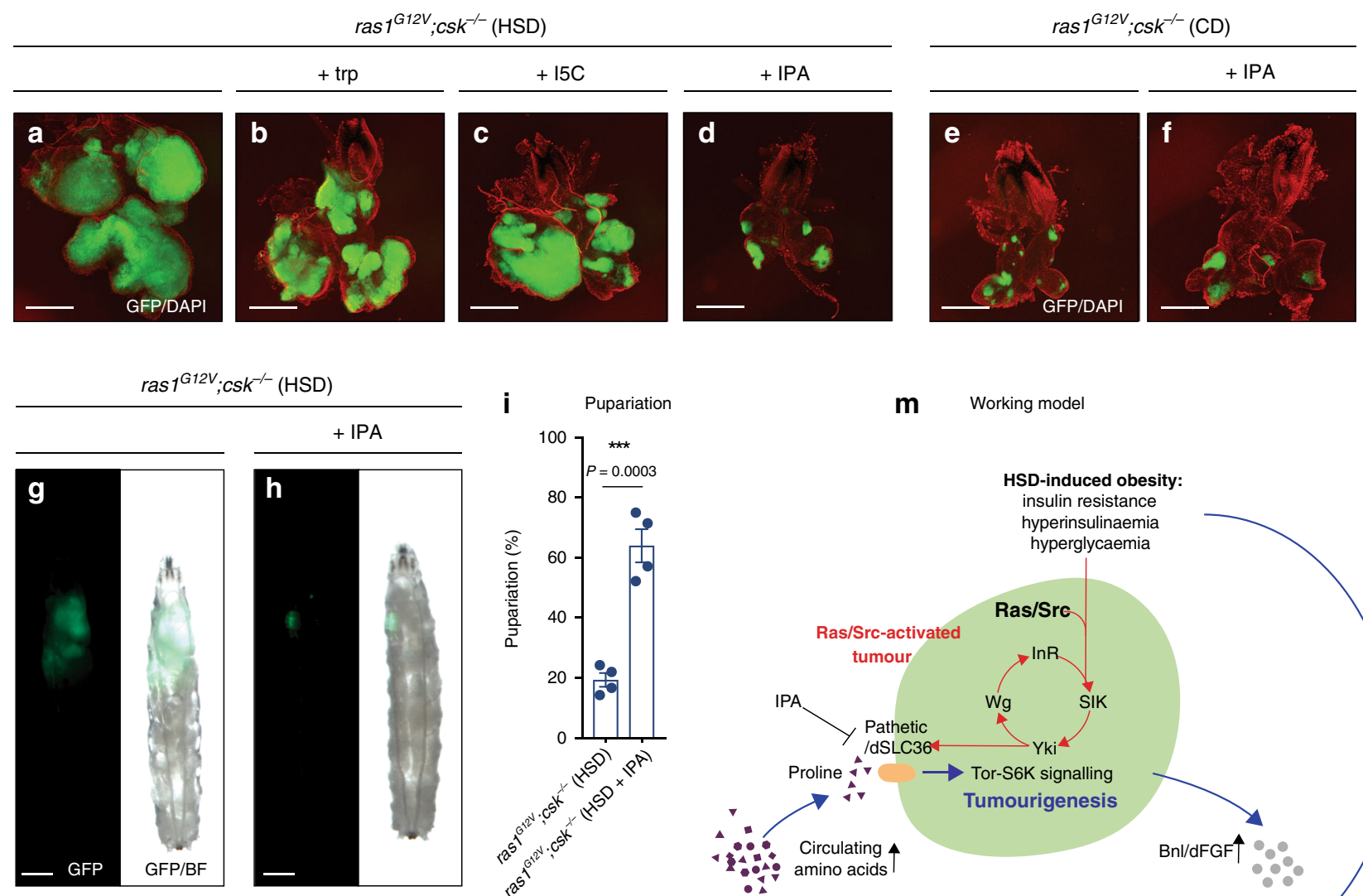

i

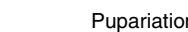

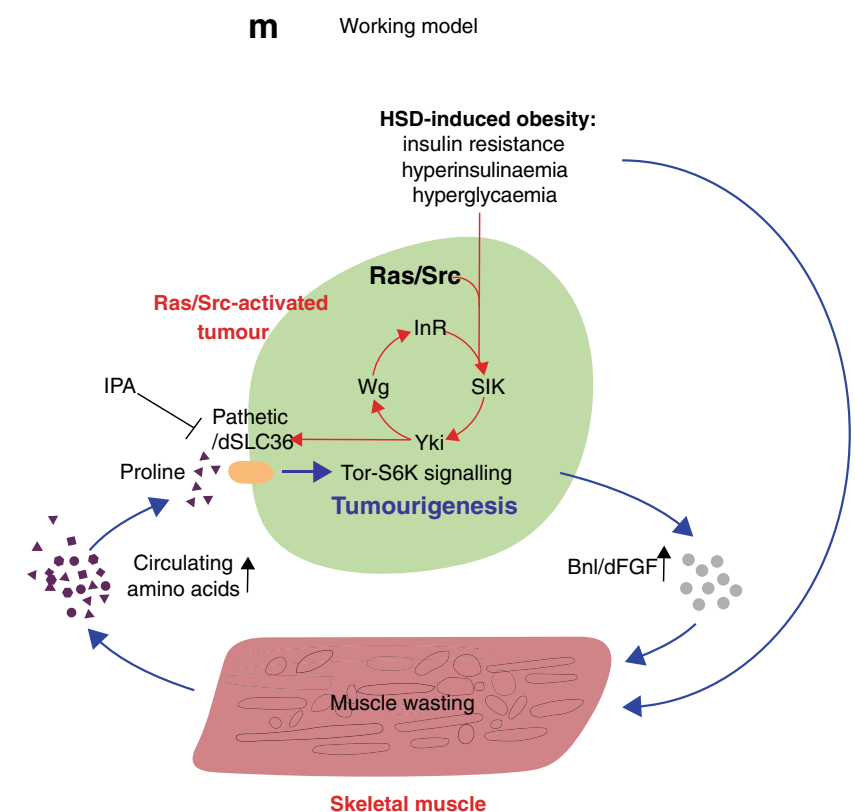

$+100 \mathrm{mM}$ pro

$\operatorname{ras}^{\mathrm{G} 12 \mathrm{~V}} ; \mathrm{csk}^{-/}$, path $(\mathrm{CD})$
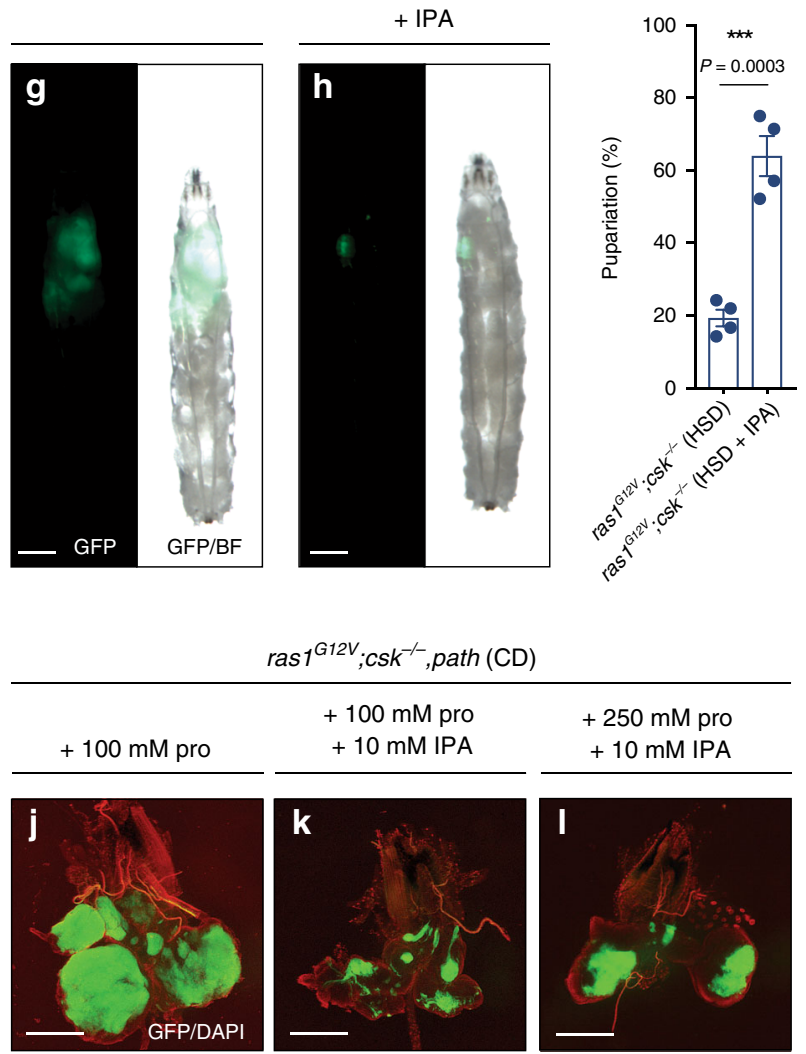

Skeletal muscle

Fig. 6 Targeting SLC36-transporter with amino acid derivative suppresses tumour growth. Dissected eye epithelial tissue stained with DAPI (red) from $\operatorname{ras}^{\mathrm{G} 12 \mathrm{~V}}$;csk ${ }^{-/}$animals raised on HSD (a), HSD supplemented with $10 \mathrm{mM}$ L-tryptophan (trp) (b), HSD supplemented with 10 mM Indole-5-carboxylic acid (I5C) (c) and HSD supplemented with $10 \mathrm{mM}$ Indole-3-propionic acid (IPA) (d). Scale bar, $250 \mu \mathrm{m}$. Dissected eye epithelial tissue stained with DAPI

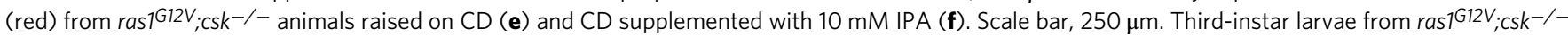
animals raised on HSD (g) and HSD supplemented with $10 \mathrm{mM} \mathrm{IPA} \mathrm{(h).} \mathrm{Scale} \mathrm{bar,} 500 \mu \mathrm{m}$. i Pupariation percentage of ras $1^{G 12 V ; \text { csk }^{-/-}}$animals raised on HSD and HSD supplemented with $10 \mathrm{mM}$ IPA. Results are shown as mean \pm SEM. Data from total $n=120$ (HSD) and $n=75$ (HSD + IPA) from four independent experiments. Data were analysed by two-tailed unpaired Student's $t$ test. Asterisks indicate statistically significant difference ( $\left.{ }^{\star \star \star} P<0.001\right)$. Dissected eye epithelial tissue stained with DAPI (red) from ras $1 \mathrm{G}^{2} \mathrm{~V}_{;}$csk $\mathrm{K}^{-/-}$, path animals raised on CD supplemented with $100 \mathrm{mM} \mathrm{L-proline} \mathrm{(j),} \mathrm{CD}$ supplemented with $100 \mathrm{mM}$ L-proline and $10 \mathrm{mM}$ IPA (k) and CD supplemented with $250 \mathrm{mM}$ L-proline and $10 \mathrm{mM}$ IPA (I). Scale bar, $250 \mu \mathrm{m}$. $\mathbf{m}$ Working model illustrating dual-layered coordination of tumour metabolic response: (1) at the whole organism level-branchless (Bnl)-dependent induction of cachexia-like muscle wasting and systemic amino acid release (blue arrows)-and (2) at the tumour-autonomous level-Yorkie-dependent induction of amino acid transporter expression (red arrows). The tumour-autonomous SIK-Yki-Wg-InR circuit was identified previously ${ }^{14}$. IPA represents a therapeutic strategy to break the nutritional circuit between muscle wasting and tumour growth by targeting the SLC36-family transporter Path. SIK Salt-inducible kinase, Yki Yorkie, Wg wingless, InR insulin receptor, IPA indole-3-propionic acid.

(1) UAS-LacZ; FRT82B; (2) UAS-ras1 G12V; FRT82B, csk 156 Stop/TM6b; (3) UASrasl $112 V ;$ FRT82B, csk Q156Stop, UAS-bnlRNAi/GD/TM6b; (4) UAS-ras1G12V; FRT82B, csk Q156Stop, UAS-bnl RNAi/TRiP/TM6b; (5) UAS-ras1 ${ }^{\text {G12V }}$; FRT82B, csk Q156Stop, UAS-

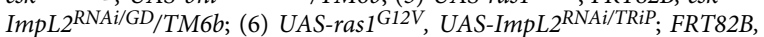
csk Q156Stop/TM6b; (7) UAS-ras1 G12V; FRT82B, csk Q156Stop, UAS-Pvf1 RNAi/GD/ TM6b; (8) UAS-ras1 $1^{G 12 V}$, UAS-Pvf1 ${ }^{R N A i / K K}$; FRT82B, csk Q156Stop/TM6b; (9) UASras1 ${ }^{\text {G12V }}$, UAS-Pvf2 ${ }^{R N A i / K K}$; FRT82B, csk Q156Stop/TM2; (10) UAS-ras1 ${ }^{\text {G12V }}$, UASPvf2 ${ }^{\text {RNAi/TRiP }}$; FRT82B, csk ${ }^{Q 156 S t o p} / T M 2$; (11) UAS-ras1 ${ }^{G 12 V}$, UAS-btl ${ }^{R N A i / G D}$; FRT82B, csk ${ }^{Q 156 S t o p} / T M 6 b$; (12) UAS-ras1 ${ }^{\text {G12V }}$, UAS-btt ${ }^{R N A i / K K} ;$ FRT82B,

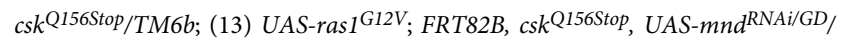
TM6b; (14) UAS-ras 1 G12V; FRT82B, cskQ156Stop, UAS-JhI-21 RNAi/GD/TM6b; (15)

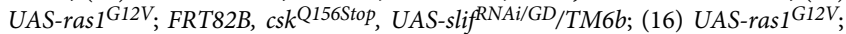
FRT82B, csk ${ }^{\text {Q156Stop, UAS-CG8785 }}{ }^{\text {RAAi/GD } / T M 6 b ; ~(17) ~ U A S-r a s 1 ~}{ }^{\text {G12V }}$, UASpath $^{R N A i / K K}$; FRT82B, csk ${ }^{Q 156 S t o p} / T M 6 b ;$; (18) UAS-ras1 $1^{G 12 V}$, UAS-path ${ }^{R N A i / T R i P}$; FRT82B, csk Q156Stop/TM6b; (19) UAS-ras1G12V; FRT82B, csk ${ }^{\mathrm{Q} 1565 t o p}$, UASCG1139RNAi/GD/TM6b; (20) UAS-ras1 G12V, CG1139RNAi/KK; FRT82B, csk ${ }^{\text {Q156Stop/ }}$

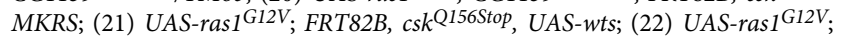
FRT82B, csk Q156Stop, UAS-yki; (23) UAS-ras1 G12V; FRT82B, csk ${ }^{\text {Q156Stop, UAS-path/ }}$ 
TM6b; (24) UAS-ras1 ${ }^{\text {G12V }}$, UAS-CG1139; FRT82B, csk ${ }^{\text {Q156Stop } / M K R S ; ~(25) ~}$ FRT82B, UAS-path; (16) UAS-CG1139; FRT82B; (26) UAS-ras1 ${ }^{\text {G12V }}$, UAS-Tor ${ }^{T E D}$; FRT82B, csk ${ }^{Q 156 S t o p} / M K R S ; ~(27) U A S-r a s 1^{G 12 V} ;$ FRT82B, UAS-S6KSTDETE, csk $^{Q 156 S t o p} / T M 6 b ;(28) U A S-r a s 1^{G 12 V}$, UAS-TorTED; FRT82B, csk ${ }^{Q 156 S t o p}$, UAS path/TM6b.

To block ecdysone signalling, we used a temperature-sensitive allele of ecdysoneless $\left(e c d^{1}\right)$. Flies with the genotype ey(3.5)-FLP1; act $>y+>$ gal4, UAS GFP; ecd ${ }^{1}$, FRT82B, tub-gal80 were crossed with UAS-ras $1^{G 12 V}$; ecd ${ }^{1}, F R T 82 B$, $c s k^{Q 156 S t o p} / T M 6 b$ flies and reared at $25^{\circ} \mathrm{C}$. At second instar larval stage (day 8 in $25^{\circ} \mathrm{C}$ ), cultures were switched to a restrictive temperature of $29^{\circ} \mathrm{C}$.

To carry out fat body-specific genetic alterations, $F B$-gal4 flies were crossed with $U A S-L a c Z$ or UAS-bnl.

To carry out muscle-specific genetic alterations, $m h c$-gal4 flies were crossed

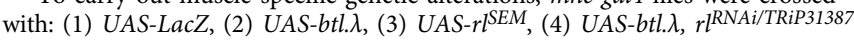
and (5) UAS-btl. $\lambda, r l^{R N A i / T R i P 31524}$

Cultures. Cultures were carried out on a modified Bloomington semi-defined medium (https://bdsc.indiana.edu/information/recipes/germanfood.html), containing sucrose $(0.15$ and $1.0 \mathrm{M}$ in $\mathrm{CD}$ and HSD, respectively) as the only purified sugar source. Ingredients were obtained from; Agar (Fisher Scientific; BP2641-1), Brewer's Yeast (MP Biomedicals; 903312, Lot: BCBN0171V), Yeast Extract (SigmaAldrich; 70161), Peptone (Sigma-Aldrich; 82303), Sucrose (Fisher Scientific; S/ 8560/63), Magnesium sulfate hexahydrate (Fluka; 00627), Calcium chloride dihydrate (Sigma-Aldrich; 223506), Propionic acid (Sigma-Aldrich; P1386), pHydroxy-benzoic acid methyl ester (Sigma-Aldrich; H5501). For dietary supplementation experiments, L-proline (Sigma-Aldrich; P5607), D-proline (SigmaAldrich; 858919), L-glycine (VWR Chemicals; 101196X), L-alanine (SigmaAldrich; 7627), L-tryptophan (Sigma-Aldrich; T0254), Indole-5-carboxylic acid (Sigma-Aldrich; I5400) and IPA (Sigma-Aldrich; 57400) were added to CD or HSD. Cultures were performed at $25^{\circ} \mathrm{C}$ unless otherwise noted. HSD feeding led to a developmental delay in reaching the third-instar larval stage; all experiments were performed on late third-instar larvae at day 8 after egg laying (CD) and day 14 after egg laying (HSD) unless otherwise noted.

Cell cultures and proliferation assay. Pancreatic cancer cell line Panc 04.03 was obtained from the American Type Culture Collection. Cells were plated in 96-well plates in a density of 2500 cells/well and cultured in RPMI, DMEM, DMEM + 1 $\mathrm{mM}$ proline, with or without $1 \mathrm{mM}$ IPA. All media was supplemented with $2 \mathrm{mM}$ glutamine, $100 \mathrm{U} / \mathrm{mL}$ penicillin/streptomycin and $10 \%$ dialysed foetal calf serum (3000 Da MWCO) in a humidified incubator at $37^{\circ} \mathrm{C}, 5 \% \mathrm{CO}_{2}$. Proliferation was measured for $120 \mathrm{~h}$ using the IncuCYTE live cell imaging system (Essen Biosciences). Datapoints were taken every $2 \mathrm{~h}$. Growth rates were calculated using the Growthcurver package in $\mathrm{R}$ (ver. 0.3.0).

Immunohistochemistry. Tissue was dissected in phosphate-buffered saline (PBS), fixed in $4 \%$ PFA/PBS for $30^{\prime}$ on ice, washed in PBS-T, incubated with primary antibody in PAXDG (PBS containing 1\% BSA, 0.3\% Triton X-100, 0.3\% deoxycholate and $5 \%$ goat serum), followed by washing and incubation with secondary antibody in PAXDG and subsequent mounting in Vectashield with DAPI (Vector Laboratories). Primary antibodies used were: rat anti-myosin (ab51098; Abcam; 1:100) and mouse anti-pERK (M-8159; Sigma; 1:50). Polyclonal rabbit antiCG1139 (1:200) was purified by New England Peptide Inc., MA, USA. An amino acid sequence $26-40$ was selected as the epitope. The following primary antibodies were kindly gifted to us: rat anti-bnl (M. Krasnow; 1:50), guinea pig anti-Path (J. Parrish; 1:200) and rabbit anti-phospho-Drosophila S6 (pS6) (A. Teleman; 1:500). Secondary antibodies used were: Alexa Fluor 488 and 568 conjugated antirat antibody (A-11006 and A-11077; Thermo Fisher Scientific; 1:200), Alexa Fluor 568 conjugated anti-mouse antibody (A-11031; Thermo Fisher Scientific; 1:200), Alexa Fluor 568 conjugated anti-rabbit antibody (A-11036; Thermo Fisher Scientific; 1:200) and Alexa-568 conjugated anti-guinea pig antibody (A-11075; Thermo Fisher Scientific, 1:200). Rhodamine phalloidin (R415; Invitrogen; 1:500) was used to visualise F-actin.

Nile red staining. Tissue was dissected in PBS, fixed in $4 \% \mathrm{PFA} / \mathrm{PBS}$ for $30^{\prime}$ on ice, incubated with $10 \mu \mathrm{g} / \mathrm{mL}$ Nile Red stain (N1142; Thermo Fisher Scientific) in PBS for $30^{\prime}$ on ice followed by washing and subsequent mounting in Vectashield with DAPI.

Acridine orange staining. Midguts were dissected in PBS on ice, incubated in 5 $\mu \mathrm{g} / \mathrm{ml}$ of acridine orange (A1301: Thermo Fisher Scientific), followed by washing and subsequent mounting in Vectashield with DAPI. Samples were imaged immediately with the Leica SP5 II confocal microscope and Leica Application Suite Advanced Fluorescence software (Leica Microsystems).

Blue dye feeding assay. Larvae were placed on food containing $1 \%$ FCF-blue dye for $90 \mathrm{~min}$ and washed in $\mathrm{dH}_{2} \mathrm{O}$ to remove excess dye from their cuticle. Larvae were imaged with Leica DFC $3000 \mathrm{G}$ digital camera to visualise blue food in the gut, or retained for feeding quantification. For feeding quantification five larvae were pooled into $1.5 \mathrm{ml}$ Eppendorf tubes each containing $70 \mu \mathrm{d} \mathrm{dH} 2 \mathrm{O}$, followed by homogenisation with motorised pestle and mortar, and centrifuged for $5 \mathrm{~min}$ at $10,000 \mathrm{RCF} .40 \mu \mathrm{l}$ of supernatant from each sample was transferred to a transparent 96-well plate and absorbance was measured at $629 \mathrm{~nm}$ in BMG Labtech FLUOstar Omega Microplate reader.

Smurf gut integrity assay. Larvae were placed on food containing $1 \%$ FCF-blue dye overnight, with a minimum of ten animals per vial. Larvae were washed in $\mathrm{dH}_{2} \mathrm{O}$ to remove excess dye from their cuticle and scored as 'Smurfs' if blue dye was only visible in the gut, or 'Non-smurfs', if blue dye was visible across the whole animal.

Muscle wasting quantification. Individual larvae were washed and dissected in PBS. Larval body-wall muscle was stained with Rhodamine phalloidin (R415; Invitrogen; 1:500) to visualise F-actin. Individual animals were scored into categories each representing the strength of muscle wasting from either 'none'; 'minor'; 'moderate' or 'strong' (Supplementary Fig. 1f-i). Scores were assigned based on wasting of the ventral longitudinal, lateral oblique and lateral longitudinal muscles centred around the fourth abdominal segment (A4) of the larval body wall and each hemisegment was used as an internal control (Supplementary Fig. 1e). A minimum of 30 animals were scored per condition. Body-wall muscle staining and quantification was performed on animals from day $8(\mathrm{CD})$, day 10 (early stage; HSD), day 14 (mid stage; HSD) and day 16 (late stage; HSD) after egg laying.

Microscopy and imaging. Larval images were acquired with Leica M165 FC fluorescent microscope equipped with Leica DFC $3000 \mathrm{G}$ digital camera. Eye disc images were acquired with Leica M165 FC fluorescent microscope equipped with S-View SXY-I30 digital camera. Confocal images were acquired with Leica SP5 II confocal microscope and Leica Application Suite Advanced Fluorescence software (ver. 4.6.1) (Leica Microsystems). Larval GFP/brightfield overlay images were compiled with Adobe Photoshop software.

Immunostaining quantification. Quantification of staining intensity for Bnl, Path CG1139 and phospho-Drosophila ribosomal protein S6 (pS6), each stained with Alexa-568 secondary antibody, was carried out on GFP-positive clones. A minimum of three samples per condition were analysed in ImageJ software (ver. 2.0.0 rc-43) using a custom-made macro written by D. Dormann. RGB images were first converted to tagged image file format (tiff), channels were split and the green channel was thresholded for GFP intensity to allow for the identification of GFPpositive clone area. For the GFP-positive clone area, mean pixel intensity was calculated across the red channel.

Eye disc area quantification. A minimum of three samples per condition were analysed in ImageJ software (ver. 2.0.0-rc-43) using a custom-made macro written by D. Dormann. Images were first converted to tiff format and RGB channels were split. The eye disc area was manually defined, based on the outline of DAPI staining in the red channel. The green channel was thresholded for GFP intensity to allow for the identification of GFP-positive clones within the user-defined eye disc. Clone area was subsequently calculated.

Larval video tracking and analysis. Larvae were maintained in normal culture conditions up until the point of video recording. Individual larvae were picked and placed onto the centre of an agar plate at room temperature and recorded immediately. Video plate recordings were carried out with use of a Teledyne DALSA Genie Nano Camera (G3-GM11-M2420) using Gecko GigE Video Recorder software (v2.0.3.1; www.visionexperts.co.uk), at 25 frames per second, ensuring a sharp contrast between the larva and background. Twenty individual larvae were recorded per condition up to a maximum of $5 \mathrm{~min}$ or until the larva was no longer in the field of view. Individual videos-each corresponding to one larva-were segmented, tracked and skeletonised using Tierpsy Tracker ${ }^{16}$. Two hundred and fifty-six defined features, previously determined to be useful in classifying behaviour in C. elegans, were extracted from the tracking data and compared between conditions ${ }^{17}$. Correction for multiple testing was applied using the Benjamini-Hochberg procedure to control the false discovery rate to $0.05^{38}$.

Quantitative RT-PCR. Larvae were washed in PBS and dissected in RNALater solution. Total RNA was extracted from pooled dissected tissue using the QIAGEN RNeasy Mini Kit. First-strand DNA was synthesised with the iScript cDNA synthesis kit (Bio-Rad). PCR was performed by mixing cDNA samples with iTaq Universal SYBR Green Supermix (Bio-Rad), ROX passive reference dye (Bio-Rad) and the relevant primers in a 96-well plate. Analysis was carried out on a 7900HT Real-Time PCR system with Applied Biosystems Software (SDS v2.4) (Applied Biosystems). Data analysis was carried out in Microsoft Excel (16.16.4). At least three independent biological replicates and two technical replicates were used. Expression values were normalised to Act88F. Primers used are listed in Supplementary Table 1 . 
RNA-sequencing. RNA from pooled dissected tissue was extracted with the QIAGEN RNeasy Mini Kit. A minimum of three samples were prepared for each genotype. RNA-seq libraries were prepared from a minimum of $10 \mathrm{ng}$ of total RNA using the Illumina Truseq mRNA stranded library prep kit (Illumina, San Diego, USA) according to the manufacturer's protocol. Library quality was checked on a Bioanalyser HS DNA chip and concentrations were estimated by Qubit measurement. Libraries were pooled in equimolar quantities and sequenced on a Hiseq2500 using paired end $100 \mathrm{bp}$ reads. At least 35 million reads passing filter were achieved per sample. After demultiplexing, raw or trimmed RNA-Seq reads were aligned against Ensembl Drosophila genome reference sequence assembly (dm3) and transcript annotations using TopHat $2^{39}$. Trim Galore, developed at The Babraham Institute by Felix Krueger (http://www.bioinformatics.babraham.ac.uk/projects/ trim_galore/), was applied for trimming adaptor and low-quality reads. For differential gene expression analysis, gene-based read counts were then obtained using the featureCounts function from Rsubread Bioconductor package ${ }^{40}$. Differential expression analysis was performed on the counts data using DESeq2 Bioconductor package $^{41}$. From tumour RNA-sequencing data, putative tumour-secreted factors were annotated based on identification of genes labelled with the GO term 'extracellular' or presence of the signal peptide sequence as identified by the signal peptide database (http://www.signalpeptide.de).

Gene set enrichment analysis. GSEA was carried out using a ranked gene list based on Wald statistics from DESeq2 results $^{42}$. MSigDB gene sets from ' $\mathrm{H}$ ', 'C2' and ' $\mathrm{C5}$ ' collections were used and only considered where FDR $q$ value $<0.25$. Normalised enrichment scores were calculated for multiple terms within functional categories. Results were displayed as cumulative normalised enrichment score, as previously published ${ }^{43}$.

Haemolymph sample preparation. Pooled haemolymph was collected from multiple animals on ice to a combined minimum sample volume of $25 \mu$. Each sample was mixed with $225 \mu \mathrm{l}$ of methanol containing internal standards (IS) $(50 \mu \mathrm{m})$. Then, chloroform $(250 \mu \mathrm{l})$ and Milli-Q water $(100 \mu \mathrm{l})$ were added, mixed and centrifuged. The water layer was filtrated through a $5-\mathrm{kDa}$ cut-off filter to remove macromolecules. Filtrate was centrifugally concentrated and resuspended in $25 \mu \mathrm{l}$ of ultrapure water prior to measurement.

Haemolymph metabolomic analyses by capillary electrophoresis mass spectrometry (CE-MS). Targeted metabolomic analyses were performed by the Human Metabolome Technologies Inc., Yamagata, Japan (HMT). CE-MS was performed using Agilent CE-TOFMS Machine (CE-TOFMS) and Agilent 6460 TripleQuad LC/MS Machine (CE-QqQMS)(Agilent Technologies). Peaks detected in CE-TOFMS analysis were extracted using automatic integration software (MasterHands ver.2.17.1.11 developed at Keio University) ${ }^{44}$ and those in CEQqQMS analysis were extracted using automatic integration software (MassHunter Quantitative Analysis B.06.00 Agilent Technologies, Santa Clara, CA, USA) in order to obtain peak information including $\mathrm{m} / \mathrm{z}$, migration time (MT) and peak area. Putative metabolites were assigned from peak alignments based on the HMT metabolite database on the basis of $\mathrm{m} / z$ and MT. Relative peak values were calculated based on IS and normalised to sample volumes. Absolute quantification was calculated by normalising the peak area of each metabolite with respect to the area of the IS and by using standard curves, which were obtained by three-point calibrations. For haemolymph samples, 214 metabolites (135 metabolites in cation mode and 79 metabolites in anion mode, respectively) were annotated based on the HMT metabolite database, including 3-methylhistidine and proline.

Sample preparation for targeted proline quantification. Frozen tissue samples (15 pooled tumours per replicate) or haemolymph samples $(1 \mu \mathrm{L}$ per replicate) were extracted with $300 \mu \mathrm{L}$ of extraction solvent (water/methanol, 20:80 v/v). The extraction solvent contained IS at $150 \mathrm{ng} / \mathrm{mL}$ using either L-proline-2,5,5-d3 (Sigma) or ${ }^{13} \mathrm{C}_{5}{ }^{15} \mathrm{~N}$-L-proline (Sigma), depending on the experiment. Samples were sonicated using an ultrasonic water bath $(15 \mathrm{~min})$ and centrifuged $(13,000 \times g$, $\left.10 \mathrm{~min}, 5^{\circ} \mathrm{C}\right)$. An aliquot of the supernatant $(180 \mu \mathrm{L})$ was then filtered into a $96-$ well V-bottom plate using a 96-well filter plate (PTFE $0.45 \mu \mathrm{m}$ ). Pooled quality controls were created for each batch by pooling equal aliquots of each study sample in the batch, in order to assess technical reproducibility.

Targeted proline quantification by LC-MS/MS. Chromatographic analyses were carried out on a Vanquish Flex Binary UHPLC system (Thermo Scientific Inc., MA, USA) coupled to a benchtop hybrid quadrupole-Orbitrap Q Exactive mass spectrometer (Thermo Scientific Inc., Bremen, Germany). Separation was achieved using an Accucore 150 Amide HILIC column (Thermo Scientific, $150 \times 2.1 \mathrm{~mm}, 2.6 \mu \mathrm{m}$ ) equipped with a guard column (Thermo Scientific, $30 \times 2.1 \mathrm{~mm}, 2.6 \mu \mathrm{m}$ ), both held at a temperature of $40^{\circ} \mathrm{C}$ and a flow rate of $0.2 \mathrm{~mL} / \mathrm{min}$. Mobile phases were $10 \mathrm{mM}$ aqueous ammonium acetate/acetonitrile $(10: 90 \mathrm{v} / \mathrm{v})($ solvent $\mathrm{A})$ and $10 \mathrm{mM}$ aqueous ammonium acetate/acetonitrile/water $(10: 10: 80 \mathrm{v} / \mathrm{v})($ solvent B). The gradient elution was performed with a $25-47 \%$ solvent B gradient over $14 \mathrm{~min}$, held at $47 \% \mathrm{~B}$ for $4 \mathrm{~min}$ and returning to $25 \% \mathrm{~B}$ at $20 \mathrm{~min}$. The column was equilibrated for 10 min, yielding a total run time of $30 \mathrm{~min}$. Ionisation was performed in the positive ionisation mode using a heated electrospray ionisation source, with the following parameters: spray voltage $3.0 \mathrm{KV}$, heater temperature $330^{\circ} \mathrm{C}$, capillary temperature $320^{\circ} \mathrm{C}$, S-lens RF level 50, sheath and auxiliary gas flow rate, 35 and 10 units, respectively. The mass accuracy was calibrated prior to sample analysis. Mass spectrometric data were acquired in profile mode using the parallel reaction monitoring method with compound-specific settings defined in the inclusion list (Supplementary Table 2). Further acquisition settings were MS2 resolution of 17,500 (at $m / z 200$ ) and an isolation window of $m / z$ 1.2. Nitrogen was utilised as collision gas in the higher energy collisional dissociation cell with normalised collision energy as summarised in Supplementary Table 2. Automatic gain control was set to 1e5 and maximum injection time $100 \mathrm{~ms}$. Xcalibur version 4.1 was used for data acquisition and processing. Specific fragment ions were used in the data processing in order to detect and quantify each compound unequivocally. Relative quantification is reported based on the peak area ratio of the analyte and the respective IS.

Statistics and reproducibility. Unless otherwise stated, all statistical analyses were carried out with an unpaired, two-tailed Student's $t$ test in GraphPad Prism software (v.7.0d) where $P \leq 0.0001=* * * * ; 0.0001<P \leq 0.001=* * * ; 0.001<P \leq 0.01$ $=* * ; 0.01<P \leq 0.05=* ; P>0.05=$ not significant. All experiments were independently repeated at least three times.

Reporting summary. Further information on research design is available in the Nature Research Reporting Summary linked to this article.

\section{Data availability}

All raw RNA-sequencing data that support the findings of this study have been deposited in the GEO repository with the accession codes GSE139958. Other data that support the study are available from the corresponding author upon reasonable request.

Received: 7 July 2020; Accepted: 13 August 2020;

Published online: 16 September 2020

\section{References}

1. Hosios, A. M. et al. Amino acids rather than glucose account for the majority of cell mass in proliferating mammalian cells. Dev. Cell 36, 540-549 (2016).

2. Jain, M. et al. Metabolite profiling identifies a key role for glycine in rapid cancer cell proliferation. Science 336, 1040-1044 (2012).

3. Sahu, N. et al. Proline starvation induces unresolved ER stress and hinders mTORC1-dependent tumorigenesis. Cell Metab. 24, 753-761 (2016).

4. Olivares, O. et al. Collagen-derived proline promotes pancreatic ductal adenocarcinoma cell survival under nutrient limited conditions. Nat. Commun. 8, 16031 (2017).

5. Commisso, C. et al. Macropinocytosis of protein is an amino acid supply route in Ras-transformed cells. Nature 497, 633-637 (2013).

6. Fearon, K. C., Glass, D. J. \& Guttridge, D. C. Cancer cachexia: mediators, signaling, and metabolic pathways. Cell Metab. 16, 153-166 (2012).

7. Petruzzelli, M. \& Wagner, E. F. Mechanisms of metabolic dysfunction in cancer-associated cachexia. Genes Dev. 30, 489-501 (2016).

8. Schmidt, S. F., Rohm, M., Herzig, S. \& Diaz, M. B. Cancer cachexia: more than skeletal muscle wasting. Trends Cancer 4, 849-860 (2018).

9. Kwon, Y. et al. Systemic organ wasting induced by localized expression of the secreted insulin/IGF antagonist ImpL2. Dev. Cell 33, 36-46 (2015).

10. Figueroa-Clarevega, A. \& Bilder, D. Malignant Drosophila tumors interrupt insulin signaling to induce cachexia-like wasting. Dev. Cell 33, 47-55 (2015).

11. Song, W. et al. Tumor-derived ligands trigger tumor growth and host wasting via differential MEK activation. Dev. Cell 48, 277-286 (2019).

12. Katheder, N. S. et al. Microenvironmental autophagy promotes tumour growth. Nature 541, 417-420 (2017).

13. Hirabayashi, S., Baranski, T. J. \& Cagan, R. L. Transformed Drosophila cells evade diet-mediated insulin resistance through wingless signaling. Cell 154, 664-675 (2013)

14. Hirabayashi, S. \& Cagan, R. L. Salt-inducible kinases mediate nutrient-sensing to link dietary sugar and tumorigenesis in Drosophila. Elife 4, e08501 (2015).

15. Musselman, L. P. et al. A high-sugar diet produces obesity and insulin resistance in wild-type Drosophila. Dis. Model Mech. 4, 842-849 (2011).

16. Javer, A. et al. An open-source platform for analyzing and sharing wormbehavior data. Nat. Methods 15, 645-646 (2018).

17. Javer, A., Ripoll-Sanchez, L. \& Brown, A. E. X. Powerful and interpretable behavioural features for quantitative phenotyping of Caenorhabditis elegans. Philos. Trans. R Soc. Lond. B Biol. Sci. 373, 20170375 (2018).

18. Villanueva, J. E. et al. Time-restricted feeding restores muscle function in Drosophila models of obesity and circadian-rhythm disruption. Nat. Commun. 10, 2700 (2019).

19. Garen, A., Kauvar, L. \& Lepesant, J. A. Roles of ecdysone in Drosophila development. Proc. Natl Acad. Sci. USA 74, 5099-5103 (1977). 
20. Jiang, C., Baehrecke, E. H. \& Thummel, C. S. Steroid regulated programmed cell death during Drosophila metamorphosis. Development 124, 4673-4683 (1997).

21. Grifoni, D., Sollazzo, M., Fontana, E., Froldi, F. \& Pession, A. Multiple strategies of oxygen supply in Drosophila malignancies identify tracheogenesis as a novel cancer hallmark. Sci. Rep. 5, 9061 (2015).

22. Tipton, K. D., Hamilton, D. L. \& Gallagher, I. J. Assessing the role of muscle protein breakdown in response to nutrition and exercise in humans. Sports Med. 48, 53-64 (2018).

23. Sutherland, D., Samakovlis, C. \& Krasnow, M. A. Branchless encodes a Drosophila FGF homolog that controls tracheal cell migration and the pattern of branching. Cell 87, 1091-1101 (1996).

24. Szewczyk, N. J., Peterson, B. K., Barmada, S. J., Parkinson, L. P. \& Jacobson, L. A. Opposed growth factor signals control protein degradation in muscles of Caenorhabditis elegans. EMBO J. 26, 935-943 (2007).

25. Nowak, K., Seisenbacher, G., Hafen, E. \& Stocker, H. Nutrient restriction enhances the proliferative potential of cells lacking the tumor suppressor PTEN in mitotic tissues. Elife 2, e00380 (2013).

26. Lin, W. Y. et al. The SLC36 transporter pathetic is required for extreme dendrite growth in Drosophila sensory neurons. Genes Dev. 29, 1120-1135 (2015).

27. Ikmi, A. et al. Molecular evolution of the Yap/Yorkie proto-oncogene and elucidation of its core transcriptional program. Mol. Biol. Evol. 31, 1375-1390 (2014).

28. Thwaites, D. T. \& Anderson, C. M. The SLC36 family of proton-coupled amino acid transporters and their potential role in drug transport. $\mathrm{Br}$. J. Pharm. 164, 1802-1816 (2011).

29. Yoshida, A. et al. SLC36A1-mTORC1 signaling drives acquired resistance to CDK4/6 inhibitors. Sci. Adv. 5, eaax6352 (2019).

30. Goberdhan, D. C., Meredith, D., Boyd, C. A. \& Wilson, C. PAT-related amino acid transporters regulate growth via a novel mechanism that does not require bulk transport of amino acids. Development 132, 2365-2375 (2005).

31. Romero-Pozuelo, J., Demetriades, C., Schroeder, P. \& Teleman, A. A. CycD/ Cdk4 and discontinuities in Dpp signaling activate TORC1 in the Drosophila Wing Disc. Dev. Cell 42, 376-387.e5 (2017).

32. Metzner, L., Neubert, K. \& Brandsch, M. Substrate specificity of the amino acid transporter PAT1. Amino Acids 31, 111-117 (2006).

33. Anker, S. D. et al. Muscle wasting disease: a proposal for a new disease classification. J. Cachexia Sarcopenia Muscle 5, 1-3 (2014).

34. Toyoshima, K. et al. Increased plasma proline concentrations are associated with sarcopenia in the elderly. PLoS ONE 12, e0185206 (2017).

35. Elia, I. et al. Proline metabolism supports metastasis formation and could be inhibited to selectively target metastasizing cancer cells. Nat. Commun. 8, 15267 (2017).

36. Liu, W. et al. Proline oxidase promotes tumor cell survival in hypoxic tumor microenvironments. Cancer Res. 72, 3677-3686 (2012).

37. Loayza-Puch, F. et al. Tumour-specific proline vulnerability uncovered by differential ribosome codon reading. Nature 530, 490-494 (2016).

38. Benjamini, Y. \& Hochberg, Y. Controlling the false discovery rate-a practical and powerful approach to multiple testing. J. R. Stat. Soc. B 57, 289-300 (1995).

39. Kim, D. et al. TopHat2: accurate alignment of transcriptomes in the presence of insertions, deletions and gene fusions. Genome Biol. 14, R36 (2013).

40. Liao, Y., Smyth, G. K. \& Shi, W. featureCounts: an efficient general purpose program for assigning sequence reads to genomic features. Bioinformatics 30, 923-930 (2014).

41. Anders, S. \& Huber, W. Differential expression analysis for sequence count data. Genome Biol. 11, R106 (2010).

42. Subramanian, A. et al. Gene set enrichment analysis: a knowledge-based approach for interpreting genome-wide expression profiles. Proc. Natl Acad. Sci. USA 102, 15545-15550 (2005).

43. Cuartero, S. et al. Control of inducible gene expression links cohesin to hematopoietic progenitor self-renewal and differentiation. Nat. Immunol. 19, 932-941 (2018).
44. Sugimoto, M., Wong, D. T., Hirayama, A., Soga, T. \& Tomita, M. Capillary electrophoresis mass spectrometry-based saliva metabolomics identified oral, breast and pancreatic cancer-specific profiles. Metabolomics 6 , 78-95 (2010).

\section{Acknowledgements}

We thank members of the Hirabayashi, Miguel-Aliaga and Cochemé laboratories for helpful discussions; members of the LMS Genomics Facility (Laurence Game and Ivan Andrew) for help with RNA-sequencing; LMS Biological Mass Spectrometry and Proteomics Facility for metabolomics analysis; LMS Microcopy Facility (Dirk Dormann) for image analysis; LMS Computing and Bioinformatics Facility (Thomas Carroll and Sanjay Khadayate) for data analysis; Luigi Feriani for technical help with larval video tracking and analysis; Courtney Choutka, Julia Cordero and Irene Miguel-Aliaga for paper comments. We thank Alana O’Reilly, Michael Simon, Georg Halder, Jay Parrish, Deborah Goberdhan, Kenneth Irvine, Ronald Kühnlein, Mark Krasnow, Aurelio Teleman, the Bloomington Drosophila Stock Centre (NIH P40OD018537) and the Vienna Drosophila Resource Centre (VDRC, www.vdrc.at) for antibodies and fly strains. L.F. was supported intramural funding from the Medical Research Council (MC-A654-5QC70). A.E.X.B. was supported intramural funding from the Medical Research Council (MCA658-5TY30). S.H. was supported by grants from PRESTO, Japan Science and Technology Agency and intramural funding from the Medical Research Council (MC-A6545QC30).

\section{Author contributions}

H.N. conducted most experiments with L.C. and S.H. providing support. Y.-F. W. carried out bioinformatics analysis. J.B.M. and H.B.K. performed metabolomics experiments. A. E.X.B. conducted larval locomotion analysis. L.F. conducted human cancer cell line analysis. H.N. and S.H. conceived and designed the project, analysed data and co-wrote the paper, with all authors providing feedback.

\section{Competing interests}

The authors declare no competing interests.

\section{Additional information}

Supplementary information is available for this paper at https://doi.org/10.1038/s41467020-18502-9.

Correspondence and requests for materials should be addressed to S.H.

Peer review information Nature Communications thanks the anonymous reviewers for their contribution to the peer review of this work.

Reprints and permission information is available at http://www.nature.com/reprints

Publisher's note Springer Nature remains neutral with regard to jurisdictional claims in published maps and institutional affiliations.

Open Access This article is licensed under a Creative Commons Attribution 4.0 International License, which permits use, sharing, adaptation, distribution and reproduction in any medium or format, as long as you give appropriate credit to the original author(s) and the source, provide a link to the Creative Commons license, and indicate if changes were made. The images or other third party material in this article are included in the article's Creative Commons license, unless indicated otherwise in a credit line to the material. If material is not included in the article's Creative Commons license and your intended use is not permitted by statutory regulation or exceeds the permitted use, you will need to obtain permission directly from the copyright holder. To view a copy of this license, visit http://creativecommons.org/ licenses/by/4.0/.

(c) Crown 2020 